\title{
Multiple Criteria Decision Making Approach with Multivalued Neutrosophic Linguistic Normalized Weighted Bonferroni Mean Hamacher Operator
}

\author{
Bao-lin Li $\left(\mathbb{D},{ }^{1,2}\right.$ Juan-ru Wang ${ }^{D},{ }^{1}$ Li-hua Yang, ${ }^{2}$ and Xue-tao $\mathrm{Li}^{2}$ \\ ${ }^{1}$ School of Management, Northwestern Polytechnical University, Xian 710072, China \\ ${ }^{2}$ School of Economics and Management, Hubei University of Automotive Technology, Shiyan 442002, China \\ Correspondence should be addressed to Juan-ru Wang; wjuanru@nwpu.edu.cn
}

Received 8 August 2017; Accepted 31 December 2017; Published 18 March 2018

Academic Editor: Anna M. Gil-Lafuente

Copyright (C) 2018 Bao-lin Li et al. This is an open access article distributed under the Creative Commons Attribution License, which permits unrestricted use, distribution, and reproduction in any medium, provided the original work is properly cited.

\begin{abstract}
The neutrosophic set and linguistic term set are widely applied in recent years. Motivated by the advantages of them, we combine the multivalued neutrosophic set and linguistic set and define the concept of the multivalued neutrosophic linguistic set (MVNLS). Furthermore, Hamacher operation is an extension of the algebraic and Einstein operation. Additionally, the normalized weighted Bonferroni mean (NWBM) operator can consider the weight of each argument and capture the interrelationship of different arguments. Therefore, the combination of NWBM operator and Hamacher operation is more valuable and agile. Firstly, MVNLS and multivalued neutrosophic linguistic number (MVNLN) are defined, then some new operational rules of MVNLNs on account of Hamacher operations are developed, and the comparison functions for MVNLNs are given. Secondly, multivalued neutrosophic linguistic normalized weighted Bonferroni mean Hamacher operator (MVNLNWBMH) is proposed, and a number of expected characteristics of new operator are investigated. Meanwhile, some special cases of different parameters $p, q$, and $\varepsilon$ are analyzed. Thirdly, the approach utilizing the MVNLNWBMH operator is introduced to manage multiple criteria decision making (MCDM) issue in multivalued neutrosophic linguistic environment. Ultimately, a practical example is presented and a comparative analysis is carried out, which validate the effectiveness and generalization of the novel approach.
\end{abstract}

\section{Introduction}

In real world, due to the complexity of decision information, the fuzzy theory has attracted widespread attention and has been developed in various fields. Zadeh [1] firstly proposed the notion of fuzzy sets (FSs). Then, Atanassov [2] introduced the intuitionistic fuzzy sets (IFSs), which overcome the weakness of nonmembership degrees. Subsequently, in order to address the hesitation degree of decision makers, Torra [3] defined hesitant fuzzy sets (HFSs). Fuzzy set theory has been well promoted, but it still cannot manage the inconsistent and indeterminate information. Under this circumstance, Smarandache [4] proposed neutrosophic sets (NSs), whose indeterminacy degree is independent of both true and false membership. NS is an extension of IFS and makes decision makers express their preference more accurately, so some achievements on NSs and its extensions have been undertaken. Some various concepts of different NSs are defined. For example, Smarandache [5] and Wang et al. [6] introduced single-valued neutrosophic sets (SVNs) to facilitate its application. Ye [7] pointed out the concept of simplified neutrosophic sets (SNSs). Wang et al. [8] developed the concept of interval neutrosophic sets (INSs). However, under certain conditions, the decision makers likely give different evaluation numbers for expressing their hesitancy. Subsequently, the definition of single-valued neutrosophic hesitant fuzzy sets (SVNHFSs) was firstly proposed by Ye [9] in 2014, and then Wang and Li [10] also proposed multivalued neutrosophic sets (MVNSs) in 2015. Actually, the notions of SVNHFSs and MVNSs are equal. For simplicity, we adapt the term of MVNSs in this paper.

On the other hand, the aggregation operators, comparison method for neutrosophic numbers, have also been studied. For SVNSs, Liu and Wang [11] employed NWBM operator to solve multiple criteria problem in single-valued 
neutrosophic environment. Ye [12] gave the definitions of cross-entropy and correlation coefficient. For INSs, Zhang et al. [13] developed some aggregation operators. Liu and Shi [14] not only provided the definition of interval neutrosophic hesitant fuzzy sets (INHFSs), but also discussed the generalized hybrid weighted average operator. Broumi and Smarandache [15-17] studied the correlation coefficients, cosine similarity measure, and some new operations. Ye [18] proposed similarity measures between interval neutrosophic sets. For MVNSs, Ye [9] developed SVNHFWA and SVNHFWG operators for MCDM problem. Peng et al. $[19,20]$ extended power aggregation operators and defined some outranking relations under MVNS environment. Ji et al. [21] analyzed a novel TODIM method for MVNSs.

In real life, owing to the ambiguity of decision makers' thinking, people prefer to utilize linguistic variables for describing their assessment value rather than the quantization value. Therefore, linguistic variable has attracted widespread attention in the field of MCDM. The linguistic variable was firstly proposed by Zadeh [22] and applied for the fuzzy reasoning. After that, a series of works on it have been made. Wang et al. [23-25] presented a new approach in view of hesitant fuzzy linguistic information. Meng et al. [26] developed linguistic hesitant fuzzy sets and studied hybrid weighted operator. Tian et al. [27] defined gray linguistic weighted Bonferroni mean operator for MCDM.

In order to indicate the true, indeterminate and false extents concerning a linguistic term, the NSs and linguistic set (LS) are combined. Several neutrosophic linguistic sets and their corresponding operators are defined, for example, single-valued or simplified neutrosophic linguistic sets and trapezoid linguistic sets [28-31], interval neutrosophic certain or uncertain linguistic sets [32-34]. However, due to the hesitancy of people's thinking, the trueness of a linguistic term may be given several values, and the case is similar to the false and indeterminate extents. The existing literature does not consider this perspective. Therefore, the multivalued neutrosophic linguistic set (MVNLS) and multivalued neutrosophic linguistic number (MVNLN) in this article are proposed in order to better express the information.

Aggregation operator which can fuse multiple arguments into a single comprehensive value is an important tool for MCDM problem. Many researchers have developed some efficient operators [35-42], for instance, the weighted geometric average (WGA) or averaging (WA) operator, prioritized aggregation (PA) operator, Maclaurin symmetric mean operator, and Bonferroni mean (BM) operator. BM operator was originally defined by Bonferroni [43] and has attracted widespread attention because of its characteristics of capturing interrelationship among arguments. Some achievements have been made on it [11,44-49]. In order to aggregate neutrosophic linguistic information, some researches on aggregation operators under neutrosophic linguistic and neutrosophic uncertain linguistic environments have also been applied [28-34, 50]. Until now, BM and NWBM fail to accommodate aggregation information for multivalued neutrosophic linguistic environment. Motivated by this limitation, we will extend the NWBM operator to MVNLS in this article.

T-norms and t-conorms are two functions that satisfy certain conditions, respectively. The Archimedean t-conorms and t-norms are well known, which include algebraic,
Einstein, and Hamacher. Hamacher operation is an extension of algebraic and Einstein. Generally, the algebraic operators are common; there are also a few aggregation operations based on Einstein operations. Because Hamacher operator is more general, Liu et al. [51, 52] discussed the Hamacher operational rules. So far, there is no research for MVNLS based on Hamacher operations. Since it is better for MVNLS to depict the actual situation, NWBM operator can capture the interrelationship among arguments, and Hamacher operations are more general, it is of great meaning to study the NWBM Hamacher operators under multivalued neutrosophic linguistic environment for MCDM problems.

The main purposes of the paper are presented as follows:

(1) To better express people's hesitancy, combining the MVNS and LS, we give the notions of MVNLS and MVNLN; besides, the score, accuracy, and certainty functions are also investigated to compare MVNLNs.

(2) Due to the generalization of Hamacher operational rules, we define new operations of MVNLNs based on Hamacher operational rules and discuss their operational relations.

(3) The NWBM considering the interrelationship of different arguments has gained widespread concerns; we extend NWBM operator to MVNLN environment, the MVNLNWBMH operator is defined, and some desirable characteristics are also studied.

(4) In order to verify the effectiveness, an example for MCDM problem utilizing MVNLNWBMH operator is illustrated and a comparative analysis is conducted. We also analyze the influences of different parameter values for the final outcomes, and the results demonstrate that the operator proposed is more general and flexible.

The article is arranged in this way. In Section 2, we review a number of notions and operations for MVNS, LS, NWBM operator and Hamacher. In Section 3, we propose the definitions of MVNLS and MVNLN and develop the operations of MVNLNs on the basis of Hamacher tconorms and t-norms. Meanwhile, the algebraic as well as Einstein operations for MVNLNs are also presented, which are special cases of Hamacher operation. Moreover, the comparison method of MVNLNs is also defined. In Section 4, we propose the MVNLNWBMH operator and investigate its properties. Furthermore, when corresponding parameters are assigned different values, the special examples are also discussed. In Section 5, we establish the MCDM procedure on account of the proposed aggregation operators with MVNLS information. Section 6 presents a concrete example, and a comparison analysis is provided to show the practicability of utilizing our method. Finally, in Section 7, some results are presented.

\section{Preliminaries}

Some notions and operation are introduced in this section, which will be useful in the latter analysis.

2.1. Linguistic Term Sets. Suppose that $S=\left\{s_{1}, s_{2}, \ldots, s_{l}\right\}$ is an ordered and finite linguistic set, in which $s_{j}$ denotes a 
linguistic variable value and $\iota$ is an odd value. When $\iota$ is equal to seven, the corresponding linguistic sets are provided as follows:

$$
S=\left\{s_{1}, s_{2}, s_{3}, s_{4}, s_{5}, s_{6}, s_{7}\right\}=\{\text { extremely poor, very poor, poor, medium, good, very good, extremely good }\}
$$

In order to avoid the linguistic information loss, the set above is expanded, that is, a contiguous set, $\bar{S}=\left\{s_{\alpha} \mid \alpha \in R\right\}$.

Definition 1 (see [53]). Let $s_{i}$ and $s_{j}$ be any two linguistic variables, the corresponding operations are presented:

$$
\begin{aligned}
& \text { (1) } \lambda s_{i}=s_{\lambda \times i}, \lambda \geq 0 ; \\
& \text { (2) } s_{i} \oplus s_{j}=s_{i+j} ; \\
& \text { (3) } s_{i} \otimes s_{j}=s_{i \times j} ; \\
& \text { (4) }\left(s_{i}\right)^{\lambda}=s_{i^{\lambda}} .
\end{aligned}
$$

\subsection{Multivalued Neutrosophic Sets}

Definition 2 (see $[9,10])$. Suppose that $X$ is a collection of objects; MVNSs $A$ on $X$ is defined by

$$
A=\left\{\left\langle x, \widetilde{T}_{A}(x), \widetilde{I}_{A}(x), \widetilde{F}_{A}(x)\right\rangle \mid x \in X\right\},
$$

where $\widetilde{T}_{A}(x)=\left\{\gamma \mid \gamma \in \widetilde{T}_{A}(x)\right\}, \widetilde{I}_{A}(x)=\left\{\delta \mid \delta \in \widetilde{I}_{A}(x)\right\}$, $\widetilde{F}_{A}(x)=\left\{\eta \mid \eta \in \widetilde{F}_{A}(x)\right\}$, and $\widetilde{T}_{A}(x), \widetilde{I}_{A}(x)$, and $\widetilde{F}_{A}(x)$ are three collections of crisp numbers belonging to $[0,1]$, representing the probable true-membership degree, indeterminacy-membership degree, and falsity-membership degree, where $x$ in $X$ belongs to $A$, respectively, satisfying these conditions $0 \leq \gamma, \delta, \eta \leq 1$, and $0 \leq \sup \widetilde{T}_{A}(x)+$ $\sup \widetilde{I}_{A}(x)+\sup \widetilde{F}_{A}(x) \leq 3$. If there is only one element in $X$, $A$ is indicated by the three-tuple $A=\left\langle\widetilde{T}_{A}(x), \widetilde{I}_{A}(x), \widetilde{F}_{A}(x)\right\rangle$, that is, known as a multivalued neutrosophic number (MVNN). Generally, MVNSs are considered as the generalizations of the other sets, such as FSs, IFSs, HFSs, DHFs, and SVNSs.

\subsection{Normalized Weighted Bonferroni Mean}

Definition 3 (see [43]). Let $p, q \geq 0$ as well as $a_{i}(i=$ $1,2, \ldots, n)$ be a set of nonnegative values; then the BM is defined as

$$
\begin{aligned}
\operatorname{BM}^{p, q}\left(a_{1}, a_{2}, \ldots, a_{n}\right) \\
=\left(\frac{1}{n(n-1)} \sum_{\substack{i, j=1, i \neq j}}^{n}\left(a_{i}^{p} a_{j}^{q}\right)\right)^{1 /(p+q)} .
\end{aligned}
$$

Definition 4 (see [46]). Let $p, q \geq 0$ and $a_{i}(i=1,2, \ldots, n)$ be a set of nonnegative values, and the corresponding NWBM can be expressed as follows:

$$
\begin{aligned}
\operatorname{NWBM}^{p, q}\left(a_{1}, a_{2}, \ldots, a_{n}\right) \\
=\left(\bigoplus_{\substack{i, j=1, i \neq j}}^{n} \frac{w_{i} w_{j}}{1-w_{i}}\left(a_{i}^{p} \otimes a_{j}^{q}\right)\right)^{1 /(p+q)},
\end{aligned}
$$

where $w=\left(w_{1}, w_{2}, \ldots, w_{n}\right)$ represents the corresponding weighted vector of $a_{i}(i=1,2, \ldots, n)$, satisfying $w_{i} \geq 0$ and $\sum_{i=1}^{n} w_{i}=1$. The weight vector can be given by decision makers in real problem.

Obviously, the NWBM operator possesses a few characteristics such as commutativity, reducibility, monotonicity, boundedness, and idempotency.

2.4. Hamacher Operations. We know aggregation operator is given in accordance with different t-norms and t-conorms; there are some exceptional circumstances listed as follows:

(1) algebraic t-norm and t-conorm

$$
\begin{aligned}
& a \otimes b=a b, \\
& a \oplus b=a+b-a b ;
\end{aligned}
$$

(2) Einstein t-norm and t-conorm

$$
\begin{aligned}
& a \otimes b=\frac{a b}{1+(1-a) \times(1-b)}, \\
& a \oplus b=\frac{a+b}{1+a b}
\end{aligned}
$$

(3) Hamacher t-norm and t-conorm

$$
\begin{aligned}
& a \otimes b=\frac{a b}{\varepsilon+(1-\varepsilon)(a+b-a b)}, \\
& a \oplus b=\frac{a+b-a b-(1-\varepsilon) a b}{1-(1-\varepsilon) a b},
\end{aligned}
$$

$\varepsilon>0$.

In particular, when $\varepsilon=1, \varepsilon=2$, the algebraic and Einstein operations are the simplifications of Hamacher t-norm and $t$ conorm.

\section{Multivalued Neutrosophic Linguistic Set}

\subsection{MVNLS and Its Hamacher Operations}

Definition 5. Let $X$ be a set of points; an MVNLS $A$ in $X$ is defined as follows:

$$
A=\left\{\left\langle x,\left[s_{\theta(x)},\left(\widetilde{T}_{A}(x), \widetilde{I}_{A}(x), \widetilde{F}_{A}(x)\right)\right]\right\rangle \mid x \in X\right\},
$$


where $s_{\theta(x)} \in S, \widetilde{T}_{A}(x)=\left\{\gamma \mid \gamma \in \widetilde{T}_{A}(x)\right\}, \widetilde{I}_{A}(x)=\{\delta \mid \delta \in$ $\left.\widetilde{I}_{A}(x)\right\}, \widetilde{F}_{A}(x)=\left\{\eta \mid \eta \in \widetilde{F}_{A}(x)\right\}$, and $\widetilde{T}_{A}(x), \widetilde{I}_{A}(x)$, and $\widetilde{F}_{A}(x)$ are three sets of crisp values in $[0,1]$, denoting three degrees of $x$ in $X$ belonging to $s_{\theta(x)}$, which are trueness, indeterminacy, and falsity, satisfying these conditions $0 \leq$ $\gamma, \delta, \eta \leq 1$, and $0 \leq \sup \widetilde{T}_{A}(x)+\sup \widetilde{I}_{A}(x)+\sup \widetilde{F}_{A}(x) \leq 1$.

Definition 6. Let $A=\left\{\left\langle x,\left[s_{\theta(x)},\left(\widetilde{T}_{A}(x), \widetilde{I}_{A}(x), \widetilde{F}_{A}(x)\right)\right]\right\rangle \mid\right.$ $x \in X\}$ be an MVNLS; supposing there is only one element in $X$, then tuple $\left\langle s_{\theta(x)},\left(\widetilde{T}_{A}(x), \widetilde{I}_{A}(x), \widetilde{F}_{A}(x)\right)\right\rangle$ is depicted as a multivalued neutrosophic linguistic number (MVNLN). For simplicity, the MVNLN can also be represented as

$$
A=\left\{\left\langle s_{\theta(x)},\left(\widetilde{T}_{A}(x), \widetilde{I}_{A}(x), \widetilde{F}_{A}(x)\right)\right\rangle \mid x \in X\right\} .
$$

Definition 7. Let $a_{1}=\left\langle s_{\theta\left(a_{1}\right)},\left(\widetilde{T}\left(a_{1}\right), \widetilde{I}\left(a_{1}\right), \widetilde{F}\left(a_{1}\right)\right)\right\rangle$ and $a_{2}=$ $\left\langle s_{\theta\left(a_{2}\right)},\left(\widetilde{T}\left(a_{2}\right), \widetilde{I}\left(a_{2}\right), \widetilde{F}\left(a_{2}\right)\right)\right\rangle$ be two MVNLNs, and $\lambda>0$; then the operations of MVNLNs can be defined on the basis of Hamacher operations.

$$
\begin{aligned}
& \text { (1) } a_{1} \oplus a_{2}=\left\langle s_{\theta\left(a_{1}\right)+\theta\left(a_{2}\right)},\right. \\
& \left(\bigcup_{\gamma_{1} \in \widetilde{T}\left(a_{1}\right), \gamma_{2} \in \widetilde{T}\left(a_{2}\right)}\left\{\frac{\gamma_{1}+\gamma_{2}-\gamma_{1} \gamma_{2}-(1-\varepsilon) \gamma_{1} \gamma_{2}}{1-(1-\varepsilon) \gamma_{1} \gamma_{2}}\right\},\right. \\
& \bigcup_{\delta_{1} \in \tilde{I}\left(a_{1}\right), \delta_{2} \in \tilde{I}\left(a_{2}\right)}\left\{\frac{\delta_{1} \delta_{2}}{\varepsilon+(1-\varepsilon)\left(\delta_{1}+\delta_{2}-\delta_{1} \delta_{2}\right)}\right\}, \\
& \left.\left.\bigcup_{\eta_{1} \in \widetilde{F}\left(a_{1}\right), \eta_{2} \in \widetilde{F}\left(a_{2}\right)}\left\{\frac{\eta_{1} \eta_{2}}{\varepsilon+(1-\varepsilon)\left(\eta_{1}+\eta_{2}-\eta_{1} \eta_{2}\right)}\right\}\right)\right\rangle ; \\
& \text { (2) } a_{1} \otimes a_{2}=\left\langle s_{\theta\left(a_{1}\right) \times \theta\left(a_{2}\right)}\right. \text {, } \\
& \left(\bigcup_{\gamma_{1} \in \widetilde{T}\left(a_{1}\right), \gamma_{2} \in \widetilde{T}\left(a_{2}\right)}\left\{\frac{\gamma_{1} \gamma_{2}}{\varepsilon+(1-\varepsilon)\left(\gamma_{1}+\gamma_{2}-\gamma_{1} \gamma_{2}\right)}\right\},\right. \\
& \bigcup_{\delta_{1} \in \tilde{I}\left(a_{1}\right), \delta_{2} \in \tilde{I}\left(a_{2}\right)}\left\{\frac{\delta_{1}+\delta_{2}-\delta_{1} \delta_{2}-(1-\varepsilon) \delta_{1} \delta_{2}}{1-(1-\varepsilon) \delta_{1} \delta_{2}}\right\}, \\
& \left.\left.\bigcup_{\eta_{1} \in \widetilde{F}\left(a_{1}\right), \eta_{2} \in \widetilde{F}\left(a_{2}\right)}\left\{\frac{\eta_{1}+\eta_{2}-\eta_{1} \eta_{2}-(1-\varepsilon) \eta_{1} \eta_{2}}{1-(1-\varepsilon) \eta_{1} \eta_{2}}\right\}\right)\right\rangle ;
\end{aligned}
$$$$
\text { (3) } \lambda a_{1}=\left\langle s_{\lambda \theta\left(a_{1}\right)}\right. \text {, }
$$$$
\left(\bigcup_{\gamma_{1} \in \widetilde{T}\left(a_{1}\right)}\left\{\frac{\left(1+(\varepsilon-1) \gamma_{1}\right)^{\lambda}-\left(1-\gamma_{1}\right)^{\lambda}}{\left(1+(\varepsilon-1) \gamma_{1}\right)^{\lambda}+(\varepsilon-1)\left(1-\gamma_{1}\right)^{\lambda}}\right\}\right. \text {, }
$$$$
\bigcup_{\delta_{1} \in \tilde{I}\left(a_{1}\right)}\left\{\frac{\varepsilon \delta_{1}^{\lambda}}{\left(1+(\varepsilon-1)\left(1-\delta_{1}\right)\right)^{\lambda}+(\varepsilon-1) \delta_{1}^{\lambda}}\right\},
$$

$$
\begin{gathered}
\left.\left.\bigcup_{\eta_{1} \in \widetilde{F}\left(a_{1}\right)}\left\{\frac{\varepsilon \eta_{1}^{\lambda}}{\left(1+(\varepsilon-1)\left(1-\eta_{1}\right)\right)^{\lambda}+(\varepsilon-1) \eta_{1}{ }^{\lambda}}\right\}\right)\right\rangle ; \\
(4) a_{1}^{\lambda}=\left\langle s_{\theta^{\lambda}\left(a_{1}\right),}\right. \\
\left(\bigcup_{\gamma_{1} \in \widetilde{T}\left(a_{1}\right)}\left\{\frac{\varepsilon \gamma_{1}^{\lambda}}{\left(1+(\varepsilon-1)\left(1-\gamma_{1}\right)\right)^{\lambda}+(\varepsilon-1) \gamma_{1}^{\lambda}}\right\},\right. \\
\bigcup_{\delta_{1} \in \tilde{T}\left(a_{1}\right)}\left\{\frac{\left(1+(\varepsilon-1) \delta_{1}\right)^{\lambda}-\left(1-\delta_{1}\right)^{\lambda}}{\left(1+(\varepsilon-1) \delta_{1}\right)^{\lambda}+(\varepsilon-1)\left(1-\delta_{1}\right)^{\lambda}}\right\}, \\
\left.\left.\bigcup_{\eta_{1} \in \widetilde{F}\left(a_{1}\right)}\left\{\frac{\left(1+(\varepsilon-1) \eta_{1}\right)^{\lambda}-\left(1-\eta_{1}\right)^{\lambda}}{\left(1+(\varepsilon-1) \eta_{1}\right)^{\lambda}+(\varepsilon-1)\left(1-\eta_{1}\right)^{\lambda}}\right\}\right)\right\rangle .
\end{gathered}
$$

If $\varepsilon=1$, then the operations based on Hamacher operational rules in Definition 7 will be simplified to the Algebraic operational rules as follows:

(5) $a_{1} \oplus a_{2}=\left\langle s_{\theta\left(a_{1}\right)+\theta\left(a_{2}\right)}\right.$,

$$
\left(\bigcup_{\gamma_{1} \in \widetilde{T}\left(a_{1}\right), \gamma_{2} \in \tilde{T}\left(a_{2}\right)}\left\{\gamma_{1}+\gamma_{2}-\gamma_{1} \gamma_{2}\right\}\right. \text {, }
$$

$$
\left.\left.\bigcup_{\delta_{1} \in \tilde{I}\left(a_{1}\right), \delta_{2} \in \tilde{I}\left(a_{2}\right)}\left\{\delta_{1} \delta_{2}\right\}, \bigcup_{\eta_{1} \in \widetilde{F}\left(a_{1}\right), \eta_{2} \in \widetilde{F}\left(a_{2}\right)}\left\{\eta_{1} \eta_{2}\right\}\right)\right\rangle ;
$$

(6) $a_{1} \otimes a_{2}=\left\langle s_{\theta\left(a_{1}\right) \times \theta\left(a_{2}\right)},\left(\bigcup_{\gamma_{1} \in \widetilde{T}\left(a_{1}\right), \gamma_{2} \in \widetilde{T}\left(a_{2}\right)}\left\{\gamma_{1} \gamma_{2}\right\}\right.\right.$,

$$
\bigcup_{\delta_{1} \in \widetilde{I}\left(a_{1}\right), \delta_{2} \in \widetilde{I}\left(a_{2}\right)}\left\{\delta_{1}+\delta_{2}-\delta_{1} \delta_{2}\right\},
$$

$$
\left.\left.\bigcup_{\eta_{1} \in \tilde{F}\left(a_{1}\right), \eta_{2} \in \widetilde{F}\left(a_{2}\right)}\left\{\eta_{1}+\eta_{2}-\eta_{1} \eta_{2}\right\}\right)\right\rangle
$$

(7) $\lambda a_{1}=\left\langle s_{\lambda \theta\left(a_{1}\right)},\left(\bigcup_{\gamma_{1} \in \widetilde{T}\left(a_{1}\right)}\left\{1-\left(1-\gamma_{1}\right)^{\lambda}\right\}\right.\right.$,

$$
\left.\left.\bigcup_{\delta_{1} \in \tilde{I}\left(a_{1}\right)}\left\{\delta_{1}^{\lambda}\right\}, \bigcup_{\eta_{1} \in \widetilde{F}\left(a_{1}\right)}\left\{\eta_{1}^{\lambda}\right\}\right)\right\rangle ;
$$

(8) $a_{1}^{\lambda}=\left\langle s_{\theta^{\lambda}\left(a_{1}\right)},\left(\bigcup_{\gamma_{1} \in \widetilde{T}\left(a_{1}\right)}\left\{\gamma_{1}^{\lambda}\right\}\right.\right.$,

$$
\left.\left.\bigcup_{\delta_{1} \in \tilde{I}\left(a_{1}\right)}\left\{1-\left(1-\delta_{1}\right)^{\lambda}\right\}, \bigcup_{\eta_{1} \in \widetilde{F}\left(a_{1}\right)}\left\{1-\left(1-\eta_{1}\right)^{\lambda}\right\}\right)\right\rangle \text {. }
$$


Supposing $\widetilde{T}\left(a_{1}\right), \widetilde{I}\left(a_{1}\right), \widetilde{F}\left(a_{1}\right), \widetilde{T}\left(a_{2}\right), \widetilde{I}\left(a_{2}\right)$, and $\widetilde{F}\left(a_{2}\right)$ contain only one value, then the operations defined above can be reduced to the operations of SVNLNs based on algebraic operations proposed by Ye [28].

If $\varepsilon=2$, then the operations based on Hamacher operational rules in Definition 7 will be simplified to the Einstein operations of MVNLNs presented below:

$$
\begin{aligned}
& \text { (9) } a_{1} \oplus a_{2}=\left\langle s_{\theta\left(a_{1}\right)+\theta\left(a_{2}\right),}\right. \\
& \left(\bigcup_{\gamma_{1} \in \widetilde{T}\left(a_{1}\right), \gamma_{2} \in \widetilde{T}\left(a_{2}\right)}\left\{\frac{\gamma_{1}+\gamma_{2}}{1+\gamma_{1} \gamma_{2}}\right\},\right. \\
& \bigcup_{\delta_{1} \in \tilde{I}\left(a_{1}\right), \delta_{2} \in \tilde{I}\left(a_{2}\right)}\left\{\frac{\delta_{1} \delta_{2}}{2-\delta_{1}-\delta_{2}+\delta_{1} \delta_{2}}\right\}, \\
& \left.\left.\bigcup_{\eta_{1} \in \widetilde{F}\left(a_{1}\right), \eta_{2} \in \widetilde{F}\left(a_{2}\right)}\left\{\frac{\eta_{1} \eta_{2}}{2-\eta_{1}-\eta_{2}+\eta_{1} \eta_{2}}\right\}\right)\right\rangle ; \\
& \text { (10) } a_{1} \otimes a_{2}=\left\langle s_{\theta\left(a_{1}\right) \times \theta\left(a_{2}\right),}\right.
\end{aligned}
$$$$
\left(\bigcup_{\gamma_{1} \in \tilde{T}\left(a_{1}\right), \gamma_{2} \in \widetilde{T}\left(a_{2}\right)}\left\{\frac{\gamma_{1} \gamma_{2}}{2-\gamma_{1}-\gamma_{2}+\gamma_{1} \gamma_{2}}\right\},\right.
$$$$
\bigcup_{\delta_{1} \in \tilde{I}\left(a_{1}\right), \delta_{2} \in \widetilde{I}\left(a_{2}\right)}\left\{\frac{\delta_{1}+\delta_{2}}{1+\delta_{1} \delta_{2}}\right\},
$$$$
\left.\left.\bigcup_{\eta_{1} \in \tilde{F}\left(a_{1}\right), \eta_{2} \in \tilde{F}\left(a_{2}\right)}\left\{\frac{\eta_{1}+\eta_{2}}{1+\eta_{1} \eta_{2}}\right\}\right)\right\rangle ;
$$$$
\text { (11) } \lambda a_{1}=\left\langle s_{\lambda \theta\left(a_{1}\right)}\right. \text {, }
$$$$
\left(\bigcup_{\gamma_{1} \in \widetilde{T}\left(a_{1}\right)}\left\{\frac{\left(1+\gamma_{1}\right)^{\lambda}-\left(1-\gamma_{1}\right)^{\lambda}}{\left(1+\gamma_{1}\right)^{\lambda}+\left(1-\gamma_{1}\right)^{\lambda}}\right\},\right.
$$$$
\bigcup_{\delta_{1} \in \tilde{I}\left(a_{1}\right)}\left\{\frac{2 \delta_{1}^{\lambda}}{\left(2-\delta_{1}\right)^{\lambda}+\delta_{1}^{\lambda}}\right\} \text {, }
$$$$
\left.\left.\bigcup_{\eta_{1} \in \widetilde{F}\left(a_{1}\right)}\left\{\frac{2 \eta_{1}^{\lambda}}{\left(2-\eta_{1}\right)^{\lambda}+\eta_{1}^{\lambda}}\right\}\right)\right\rangle \text {; }
$$$$
\text { (12) } a_{1}^{\lambda}=\left\langle s_{\theta^{\lambda}\left(a_{1}\right)},\left(\bigcup_{\gamma_{1} \in \widetilde{T}\left(a_{1}\right)}\left\{\frac{2 \gamma_{1}^{\lambda}}{\left(2-\gamma_{1}\right)^{\lambda}+\gamma_{1}^{\lambda}}\right\}\right. \text {, }\right.
$$$$
\bigcup_{\delta_{1} \in \widetilde{I}\left(a_{1}\right)}\left\{\frac{\left(1+\delta_{1}\right)^{\lambda}-\left(1-\delta_{1}\right)^{\lambda}}{\left(1+\delta_{1}\right)^{\lambda}+\left(1-\delta_{1}\right)^{\lambda}}\right\} \text {, }
$$

$$
\left.\left.\bigcup_{\eta_{1} \in \widetilde{F}\left(a_{1}\right)}\left\{\frac{\left(1+\eta_{1}\right)^{\lambda}-\left(1-\eta_{1}\right)^{\lambda}}{\left(1+\eta_{1}\right)^{\lambda}+\left(1-\eta_{1}\right)^{\lambda}}\right\}\right)\right\rangle .
$$

Supposing $\widetilde{T}\left(a_{1}\right), \widetilde{I}\left(a_{1}\right), \widetilde{F}\left(a_{1}\right), \widetilde{T}\left(a_{2}\right), \widetilde{I}\left(a_{2}\right)$, and $\widetilde{F}\left(a_{2}\right)$ contain only one value, then the operations defined above can be reduced to the operations of SVNLNs based on Einstein operations.

Theorem 8. Let $a_{1}=\left\langle s_{\theta\left(a_{1}\right)},\left(\widetilde{T}\left(a_{1}\right), \widetilde{I}\left(a_{1}\right), \widetilde{F}\left(a_{1}\right)\right)\right\rangle, a_{2}=$ $\left\langle s_{\theta\left(a_{2}\right)},\left(\widetilde{T}\left(a_{2}\right), \widetilde{I}\left(a_{2}\right), \widetilde{F}\left(a_{2}\right)\right)\right\rangle$, and $a_{3}=\left\langle s_{\theta\left(a_{3}\right)},\left(\widetilde{T}\left(a_{3}\right), \widetilde{I}\left(a_{3}\right)\right.\right.$, $\left.\left.\widetilde{F}\left(a_{3}\right)\right)\right\rangle$ be any three MVNLNs, and $\lambda, \lambda_{1}, \lambda_{2}>0$; then the properties below are correct:

$$
\begin{aligned}
& \text { (1) } a_{1} \oplus a_{2}=a_{2} \oplus a_{1} \text {; } \\
& \text { (2) } a_{1} \otimes a_{2}=a_{2} \otimes a_{1} \text {; }
\end{aligned}
$$

(3) $\lambda\left(a_{1} \oplus a_{2}\right)=\lambda a_{1} \oplus \lambda a_{2}$;

(4) $\lambda_{1} a_{1} \oplus \lambda_{2} a_{1}=\left(\lambda_{1}+\lambda_{2}\right) a_{1}$;

(5) $a_{1}^{\lambda_{1}} \otimes a_{1}^{\lambda_{2}}=a_{1}^{\lambda_{1}+\lambda_{2}}$

(6) $a_{1}^{\lambda} \otimes a_{2}^{\lambda}=\left(a_{1} \otimes a_{2}\right)^{\lambda}$;

(7) $\left(a_{1} \oplus a_{2}\right) \oplus a_{3}=a_{1} \oplus\left(a_{2} \oplus a_{3}\right)$;

(8) $\left(a_{1} \otimes a_{2}\right) \otimes a_{3}=a_{1} \otimes\left(a_{2} \otimes a_{3}\right)$.

Then, (4) will be proved as follows.

Proof of (4). Since $\lambda_{1}, \lambda_{2}>0$,

$\lambda_{1} a_{1} \oplus \lambda_{2} a_{1}$

$=\left\langle s_{\lambda_{1} \theta\left(a_{1}\right)+\lambda_{2} \theta\left(a_{1}\right)}\right.$,

$\left(\bigcup_{\gamma_{1} \in \widetilde{T}\left(a_{1}\right)}\left\{\left(\frac{\left(1+(\varepsilon-1) \gamma_{1}\right)^{\lambda_{1}}-\left(1-\gamma_{1}\right)^{\lambda_{1}}}{\left(1+(\varepsilon-1) \gamma_{1}\right)^{\lambda_{1}}+(\varepsilon-1)\left(1-\gamma_{1}\right)^{\lambda_{1}}}\right.\right.\right.$

$+\frac{\left(1+(\varepsilon-1) \gamma_{1}\right)^{\lambda_{2}}-\left(1-\gamma_{1}\right)^{\lambda_{2}}}{\left(1+(\varepsilon-1) \gamma_{1}\right)^{\lambda_{2}}+(\varepsilon-1)\left(1-\gamma_{1}\right)^{\lambda_{2}}}$

$-\frac{\left(1+(\varepsilon-1) \gamma_{1}\right)^{\lambda_{1}}-\left(1-\gamma_{1}\right)^{\lambda_{1}}}{\left(1+(\varepsilon-1) \gamma_{1}\right)^{\lambda_{1}}+(\varepsilon-1)\left(1-\gamma_{1}\right)^{\lambda_{1}}}$

$\cdot \frac{\left(1+(\varepsilon-1) \gamma_{1}\right)^{\lambda_{2}}-\left(1-\gamma_{1}\right)^{\lambda_{2}}}{\left(1+(\varepsilon-1) \gamma_{1}\right)^{\lambda_{2}}+(\varepsilon-1)\left(1-\gamma_{1}\right)^{\lambda_{2}}}$

$-(1-\varepsilon) \frac{\left(1+(\varepsilon-1) \gamma_{1}\right)^{\lambda_{1}}-\left(1-\gamma_{1}\right)^{\lambda_{1}}}{\left(1+(\varepsilon-1) \gamma_{1}\right)^{\lambda_{1}}+(\varepsilon-1)\left(1-\gamma_{1}\right)^{\lambda_{1}}}$

$\left.\cdot \frac{\left(1+(\varepsilon-1) \gamma_{1}\right)^{\lambda_{2}}-\left(1-\gamma_{1}\right)^{\lambda_{2}}}{\left(1+(\varepsilon-1) \gamma_{1}\right)^{\lambda_{2}}+(\varepsilon-1)\left(1-\gamma_{1}\right)^{\lambda_{2}}}\right)$ 


$$
\begin{aligned}
& \cdot\left(1-(1-\varepsilon) \frac{\left(1+(\varepsilon-1) \gamma_{1}\right)^{\lambda_{1}}-\left(1-\gamma_{1}\right)^{\lambda_{1}}}{\left(1+(\varepsilon-1) \gamma_{1}\right)^{\lambda_{1}}+(\varepsilon-1)\left(1-\gamma_{1}\right)^{\lambda_{1}}}\right. \\
& \left.\left.\frac{\left(1+(\varepsilon-1) \gamma_{1}\right)^{\lambda_{2}}-\left(1-\gamma_{1}\right)^{\lambda_{2}}}{\left(1+(\varepsilon-1) \gamma_{1}\right)^{\lambda_{2}}+(\varepsilon-1)\left(1-\gamma_{1}\right)^{\lambda_{2}}}\right)^{-1}\right\} \\
& \bigcup_{\delta_{1} \in \widetilde{I}\left(a_{1}\right)}\left\{\left(\frac{\varepsilon \delta_{1}^{\lambda_{1}}}{\left(1+(\varepsilon-1)\left(1-\delta_{1}\right)\right)^{\lambda_{1}}+(\varepsilon-1) \delta_{1}^{\lambda_{1}}}\right.\right. \\
& \left.\cdot \frac{\varepsilon \delta_{1}^{\lambda_{2}}}{\left(1+(\varepsilon-1)\left(1-\delta_{1}\right)\right)^{\lambda_{2}}+(\varepsilon-1) \delta_{1}^{\lambda_{2}}}\right) \\
& \cdot\left(\varepsilon+(1-\varepsilon)\left(\frac{\varepsilon \delta_{1}^{\lambda_{1}}}{\left(1+(\varepsilon-1)\left(1-\delta_{1}\right)\right)^{\lambda_{1}}+(\varepsilon-1) \delta_{1}^{\lambda_{1}}}\right.\right. \\
& +\frac{\varepsilon \delta_{1}^{\lambda_{2}}}{\left(1+(\varepsilon-1)\left(1-\delta_{1}\right)\right)^{\lambda_{2}}+(\varepsilon-1) \delta_{1}^{\lambda_{2}}} \\
& -\frac{\varepsilon \delta_{1}^{\lambda_{1}}}{\left(1+(\varepsilon-1)\left(1-\delta_{1}\right)\right)^{\lambda_{1}}+(\varepsilon-1) \delta_{1}^{\lambda_{1}}} \\
& \left.\left.\left.\cdot \frac{\varepsilon \delta_{1}^{\lambda_{2}}}{\left(1+(\varepsilon-1)\left(1-\delta_{1}\right)\right)^{\lambda_{2}}+(\varepsilon-1) \delta_{1}^{\lambda_{2}}}\right)\right)^{-1}\right\} \\
& \bigcup_{\eta_{1} \in \widetilde{F}\left(a_{1}\right)}\left\{\left(\frac{\varepsilon \eta_{1}^{\lambda_{1}}}{\left(1+(\varepsilon-1)\left(1-\eta_{1}\right)\right)^{\lambda_{1}}+(\varepsilon-1) \eta_{1} \lambda_{1}}\right.\right. \\
& \left.\cdot \frac{\varepsilon \eta_{1}^{\lambda_{2}}}{\left(1+(\varepsilon-1)\left(1-\eta_{1}\right)\right)^{\lambda_{2}}+(\varepsilon-1) \eta_{1} \lambda_{2}}\right) \\
& \cdot\left(\varepsilon+(1-\varepsilon)\left(\frac{\varepsilon \eta_{1}^{\lambda_{1}}}{\left(1+(\varepsilon-1)\left(1-\eta_{1}\right)\right)^{\lambda_{1}}+(\varepsilon-1) \eta_{1}^{\lambda_{1}}}\right.\right. \\
& +\frac{\varepsilon \eta_{1}^{\lambda_{2}}}{\left(1+(\varepsilon-1)\left(1-\eta_{1}\right)\right)^{\lambda_{2}}+(\varepsilon-1) \eta_{1}^{\lambda_{2}}} \\
& -\frac{\varepsilon \eta_{1}^{\lambda_{1}}}{\left(1+(\varepsilon-1)\left(1-\eta_{1}\right)\right)^{\lambda_{1}}+(\varepsilon-1) \eta_{1}^{\lambda_{1}}} \\
& \left.\left.\left.\left.\left.\cdot \frac{\varepsilon \eta_{1}^{\lambda_{2}}}{\left(1+(\varepsilon-1)\left(1-\eta_{1}\right)\right)^{\lambda_{2}}+(\varepsilon-1) \eta_{1}{ }^{\lambda_{2}}}\right)\right)^{-1}\right\}\right)\right\rangle \\
& =\left\langle s_{\left(\lambda_{1}+\lambda_{2}\right) \theta\left(a_{1}\right)},\right. \\
& \left(\bigcup_{\gamma_{1} \in \widetilde{T}\left(a_{1}\right)}\left\{\frac{\left(1+(\varepsilon-1) \gamma_{1}\right)^{\lambda_{1}+\lambda_{2}}-\left(1-\gamma_{1}\right)^{\lambda_{1}+\lambda_{2}}}{\left(1+(\varepsilon-1) \gamma_{1}\right)^{\lambda_{1}+\lambda_{2}}+(\varepsilon-1)\left(1-\gamma_{1}\right)^{\lambda_{1}+\lambda_{2}}}\right\}\right. \text {, } \\
& \bigcup_{\delta_{1} \in \widetilde{I}\left(a_{1}\right)}\left\{\frac{\varepsilon \delta_{1}^{\lambda_{1}+\lambda_{2}}}{\left(1+(\varepsilon-1)\left(1-\delta_{1}\right)\right)^{\lambda_{1}+\lambda_{2}}+(\varepsilon-1) \delta_{1}^{\lambda_{1}+\lambda_{2}}}\right\},
\end{aligned}
$$

$$
\begin{aligned}
& \left.\left.\bigcup_{\eta_{1} \in \widetilde{F}\left(a_{1}\right)}\left\{\frac{\varepsilon \eta_{1}^{\lambda_{1}+\lambda_{2}}}{\left(1+(\varepsilon-1)\left(1-\eta_{1}\right)\right)^{\lambda_{1}+\lambda_{2}}+(\varepsilon-1) \eta_{1} \lambda_{1}+\lambda_{2}}\right\}\right)\right\rangle \\
& =\left(\lambda_{1}+\lambda_{2}\right) a_{1} .
\end{aligned}
$$

Therefore, (4) $\lambda_{1} a_{1} \oplus \lambda_{2} a_{1}=\left(\lambda_{1}+\lambda_{2}\right) a_{1}$ can be obtained.

Similarly, the other equations in Theorem 8 are easily certified in the light of Definition 7.

3.2. Comparison Method. The score, accuracy, and certainty functions are important indexes to rank MVNLNs, and their corresponding definition is given below.

Definition 9. Let $a=\left\langle s_{\theta(a)},(\widetilde{T}(a), \widetilde{I}(a), \widetilde{F}(a))\right\rangle$ be an MVNLN, and the score, accuracy, and certainty functions are achieved as below.

$$
\begin{aligned}
& \text { (1) } E(a)=\left(\frac{1}{l_{\widetilde{T}(a)} \mathfrak{I}(a)^{\imath} \widetilde{F}(a)}\right. \\
& \left.\sum_{\gamma \in \widetilde{T}(a), \delta \in \widetilde{I}(a), \eta \in \widetilde{F}(a)}\left(\frac{\gamma+1-\delta+1-\eta}{3}\right)\right) s_{\theta(a)}
\end{aligned}
$$

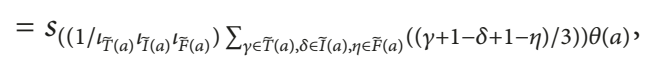

$$
\begin{aligned}
& \text { (2) } H(a)=\left(\frac{1}{l_{\widetilde{T}(a)}{ }^{l} \widetilde{F}(a)} \sum_{\gamma \in \widetilde{T}(a), \eta \in \widetilde{F}(a)}(\gamma-\eta)\right) s_{\theta(a)}
\end{aligned}
$$

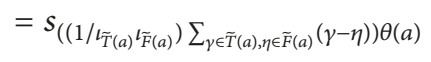

$$
\begin{aligned}
& \text { (3) } \left.C(a)=\left(\frac{1}{l_{\widetilde{T}(a)}} \sum_{\gamma \in \widetilde{T}(a)} \gamma\right) s_{\theta(a)}=s_{\left(\left(1 / \iota_{\widetilde{T}(a)}\right)\right.} \sum_{\gamma \in \widetilde{T}(a)} \gamma\right) \theta(a) \text {, }
\end{aligned}
$$

where $\iota_{\widetilde{T}(a)}, \iota_{\widetilde{I}(a)}$, and $\iota_{\widetilde{F}(a)}$ are the numbers of the values in $\widetilde{T}_{A}(x), \widetilde{I}_{A}(x)$, and $\widetilde{F}_{A}(x)$, respectively.

The linguistic variable $s_{\theta(a)}$ is important for an MVNLN. Therefore, the comparison functions defined above in Definition 9 are denoted as the linguistic variable. The bigger the truth degree $\widetilde{T}(a)$ concerning the variable $s_{\theta(a)}$ is, the smaller the indeterminacy degree $\widetilde{I}(a)$ and the false degree $\widetilde{F}(a)$ concerning the linguistic variable $s_{\theta(a)}$ are, and the higher the MVNLN is. Regarding the function of score, the greater the $\gamma-\delta-\eta$ corresponding to $s_{\theta(a)}$ is, the higher the affirmative statement is. Regarding the function of accuracy, the greater the $\gamma$ minus $\eta$ is, the more certain the statement is. Regarding the function of certainty, the bigger the $\gamma$ is, the more certain the statement is.

Based on Definition 9, the comparison method between MVNLNs is obtained.

Definition 10. Supposing $a_{1}$ and $a_{2}$ are two MVNLNs, the compared approach is achieved as follows:

(1) Supposing that $E\left(a_{1}\right)>E\left(a_{2}\right)$, then $a_{1}$ is greater than $a_{2}$, represented as $a_{1}>a_{2}$. 
(2) Supposing that $E\left(a_{1}\right)=E\left(a_{2}\right)$, and $H\left(a_{1}\right)>H\left(a_{2}\right)$, then $a_{1}$ is greater than $a_{2}$, represented as $a_{1}>a_{2}$.

(3) Supposing that $E\left(a_{1}\right)=E\left(a_{2}\right), H\left(a_{1}\right)=H\left(a_{2}\right)$, and $C\left(a_{1}\right)>C\left(a_{2}\right)$, then $a_{1}$ is greater than $a_{2}$, represented as $a_{1}>a_{2}$.

(4) Supposing that $E\left(a_{1}\right)=E\left(a_{2}\right), H\left(a_{1}\right)=H\left(a_{2}\right)$, and $C\left(a_{1}\right)=C\left(a_{2}\right)$, then $a_{1}$ equals $a_{2}$, represented as $a_{1} \sim$ $a_{2}$.

\section{The Multivalued Neutrosophic Linguistic Normalized Weighted Bonferroni Mean Hamacher Operator}

The NWBM operator not only can take into account the advantages of BM and WBM, but also has the property of reducibility and idempotency. However, the NWBM operator has not been applied to the cases where the input arguments are MVNLNs.

Definition 11. Let $a_{i}(i=1,2, \ldots, n)$ be a space of MVNLNs, $a_{i}=\left\langle s_{\theta\left(a_{i}\right)},\left(\widetilde{T}\left(a_{i}\right), \widetilde{I}\left(a_{i}\right), \widetilde{F}\left(a_{i}\right)\right)\right\rangle, p, q \geq 0$, and $\omega=\left(\omega_{1}, \omega_{2}\right.$, $\left.\ldots, \omega_{n}\right)$ be the weighted vector for $a_{i}, \omega_{i} \in[0,1]$ and $\sum_{i=1}^{n} \omega_{i}=$ 1 . Then the operator of MVNLNWBMH is achieved as below, the aggregation result is still an MVNLN.

$$
\operatorname{MVNLNWBMH}\left(a_{1}, a_{2}, \ldots, a_{n}\right)
$$

$$
=\left(\bigoplus_{\substack{i, j=1 \\ i \neq j}}^{n} \frac{\omega_{i} \omega_{j}}{1-\omega_{i}}\left(a_{i}^{p} \otimes a_{j}^{q}\right)\right)^{1 /(p+q)} .
$$

According to the operational laws in Definition 7, the results are derived below:

$$
\begin{aligned}
& \operatorname{MVNLNWBMH}\left(a_{1}, a_{2}, \ldots, a_{n}\right)=\left\langle s_{\substack{i, j=1 \\
i \neq j}}^{\left.n\left(\left(\omega_{i} \omega_{j} /\left(1-\omega_{i}\right)\right) \cdot \theta^{p}\left(a_{i}\right) \cdot \theta^{q}\left(a_{j}\right)\right)\right)^{1 /(p+q)},}\right. \\
& \left(\bigcup_{\gamma_{i} \in \widetilde{T}\left(a_{i}\right), \gamma_{j} \in \widetilde{T}\left(a_{j}\right)}\left\{\varepsilon\left(\frac{x-y}{x+(\varepsilon-1) y}\right)^{1 /(p+q)}\left(\left(1+(\varepsilon-1)\left(1-\frac{x-y}{x+(\varepsilon-1) y}\right)\right)^{1 /(p+q)}+(\varepsilon-1)\left(\frac{x-y}{x+(\varepsilon-1) y}\right)^{1 /(p+q)}\right)^{-1}\right\},\right. \\
& \bigcup_{\delta_{i} \in \tilde{I}\left(a_{i}\right), \delta_{j} \in \tilde{I}\left(a_{j}\right)}\left\{\left(\left(1+(\varepsilon-1) \frac{h}{g+(\varepsilon-1) h}\right)^{1 /(p+q)}-\left(1-\frac{h}{g+(\varepsilon-1) h}\right)^{1 /(p+q)}\right)\left(\left(1+(\varepsilon-1) \frac{h}{g+(\varepsilon-1) h}\right)^{1 /(p+q)}+(\varepsilon-1)\left(1-\frac{h}{g+(\varepsilon-1) h}\right)^{1 /(p+q)}\right)^{-1}\right\}, \\
& \left.\left.\bigcup_{\eta_{i} \in \tilde{F}\left(a_{i}\right), \eta_{j} \in \tilde{F}\left(a_{j}\right)}\left\{\left(\left(1+(\varepsilon-1) \frac{u}{v+(\varepsilon-1) u}\right)^{1 /(p+q)}-\left(1-\frac{u}{v+(\varepsilon-1) u}\right)^{1 /(p+q)}\right)\left(\left(1+(\varepsilon-1) \frac{u}{v+(\varepsilon-1) u}\right)^{1 /(p+q)}+(\varepsilon-1)\left(1-\frac{u}{v+(\varepsilon-1) u}\right)^{1 /(p+q)}\right)^{-1}\right\}\right)\right\rangle \\
& =\left\langles _ { ( \sum _ { \substack { i , j = 1 \\
i \neq j } } ^ { n } ( ( \omega _ { i } \omega _ { j } / ( 1 - \omega _ { i } ) ) \cdot \theta ^ { p } ( a _ { i } ) \cdot \theta ^ { q } ( a _ { j } ) ) ) ^ { 1 / ( p + q ) ) } } \left(\bigcup_{\gamma_{i} \in \tilde{T}\left(a_{i}\right), \gamma_{j} \in \tilde{T}\left(a_{j}\right)}\left\{\frac{\varepsilon(x-y)^{1 /(p+q)}}{\left(x+\left(\varepsilon^{2}-1\right) y\right)^{1 /(p+q)}+(\varepsilon-1)(x-y)^{1 /(p+q)}}\right\},\right.\right. \\
& \left.\left.\bigcup_{\delta_{i} \in \tilde{I}\left(a_{i}\right), \delta_{j} \in \tilde{I}\left(a_{j}\right)}\left\{\frac{\left(g+\left(\varepsilon^{2}-1\right) h\right)^{1 /(p+q)}-(g-h)^{1 /(p+q)}}{\left(g+\left(\varepsilon^{2}-1\right) h\right)^{1 /(p+q)}+(\varepsilon-1)(g-h)^{1 /(p+q)}}\right\}, \bigcup_{\eta_{i} \in \tilde{F}\left(a_{i}\right), \eta_{j} \in \tilde{F}\left(a_{j}\right)}\left\{\frac{\left(v+\left(\varepsilon^{2}-1\right) u\right)^{1 /(p+q)}-(v-u)^{1 /(p+q)}}{\left(v+\left(\varepsilon^{2}-1\right) u\right)^{1 /(p+q)}+(\varepsilon-1)(v-u)^{1 /(p+q)}}\right\}\right)\right\rangle,
\end{aligned}
$$

where

$$
\begin{aligned}
x= & \prod_{\substack{i, j=1 \\
i \neq j}}^{n}\left(\left(\varepsilon-(\varepsilon-1) \gamma_{i}\right)^{p}\left(\varepsilon-(\varepsilon-1) \gamma_{j}\right)^{q}\right. \\
& \left.+\left(\varepsilon^{2}-1\right) \gamma_{i}^{p} \gamma_{j}^{q}\right)^{\omega_{i} \omega_{j} /\left(1-\omega_{i}\right)}, \\
y= & \prod_{\substack{i, j=1 \\
i \neq j}}^{n}\left(\left(\varepsilon-(\varepsilon-1) \gamma_{i}\right)^{p}\left(\varepsilon-(\varepsilon-1) \gamma_{j}\right)^{q}\right. \\
& \left.-\gamma_{i}^{p} \gamma_{j}^{q}\right)^{\omega_{i} \omega_{j} /\left(1-\omega_{i}\right)}, \\
g= & \prod_{\substack{i, j=1 \\
i \neq j}}^{n}\left(\left(1+(\varepsilon-1) \delta_{i}\right)^{p}\left(1+(\varepsilon-1) \delta_{j}\right)^{q}\right. \\
& \left.+\left(\varepsilon^{2}-1\right)\left(1-\delta_{i}\right)^{p}\left(1-\delta_{j}\right)^{q}\right)^{\omega_{i} \omega_{j} /\left(1-\omega_{i}\right)},
\end{aligned}
$$


Proof. According to the operational rules for MVNLNs, the results below can be gained

$$
\begin{aligned}
& a_{i}^{p}=\left\langle s_{\theta^{p}\left(a_{i}\right)},\left(\bigcup_{\gamma_{i} \in \widetilde{T}\left(a_{i}\right)}\left\{\frac{\varepsilon \cdot \gamma_{i}^{p}}{\left(1+(\varepsilon-1)\left(1-\gamma_{i}\right)\right)^{p}+(\varepsilon-1) \gamma_{i}^{p}}\right\}, \bigcup_{\delta_{i} \in \widetilde{I}\left(a_{i}\right)}\left\{\frac{\left(1+(\varepsilon-1) \delta_{i}\right)^{p}-\left(1-\delta_{i}\right)^{p}}{\left(1+(\varepsilon-1) \delta_{i}\right)^{p}+(\varepsilon-1)\left(1-\delta_{i}\right)^{p}}\right\},\right.\right. \\
& \left.\left.\bigcup_{\eta_{i} \in \widetilde{F}\left(a_{i}\right)}\left\{\frac{\left(1+(\varepsilon-1) \eta_{i}\right)^{p}-\left(1-\eta_{i}\right)^{p}}{\left(1+(\varepsilon-1) \eta_{i}\right)^{p}+(\varepsilon-1)\left(1-\eta_{i}\right)^{p}}\right\}\right)\right\rangle \\
& a_{j}^{q}=\left\langle s_{\theta^{q}\left(a_{j}\right)},\left(\bigcup_{\gamma_{j} \in \widetilde{T}\left(a_{j}\right)}\left\{\frac{\varepsilon \cdot \gamma_{j}^{q}}{\left(1+(\varepsilon-1)\left(1-\gamma_{j}\right)\right)^{q}+(\varepsilon-1) \gamma_{j}^{q}}\right\}, \bigcup_{\delta_{j} \in \widetilde{I}\left(a_{j}\right)}^{\bigcup}\left\{\frac{\left(1+(\varepsilon-1) \delta_{j}\right)^{q}-\left(1-\delta_{j}\right)^{q}}{\left(1+(\varepsilon-1) \delta_{j}\right)^{q}+(\varepsilon-1)\left(1-\delta_{j}\right)^{q}}\right\},\right.\right. \\
& \left.\left.\bigcup_{\eta_{j} \in \widetilde{F}\left(a_{j}\right)}\left\{\frac{\left(1+(\varepsilon-1) \eta_{j}\right)^{q}-\left(1-\eta_{j}\right)^{q}}{\left(1+(\varepsilon-1) \eta_{j}\right)^{q}+(\varepsilon-1)\left(1-\eta_{j}\right)^{q}}\right\}\right)\right\rangle \\
& a_{i}^{p} \otimes a_{j}^{q}=\left\langle s_{\theta^{p}\left(a_{i}\right) \theta^{q}\left(a_{j}\right)},\left(\bigcup_{\gamma_{i} \in \widetilde{T}\left(a_{i}\right), \gamma_{j} \in \widetilde{T}\left(a_{j}\right)}\left\{\frac{\varepsilon \gamma_{i}^{p} \gamma_{j}^{q}}{\left(\varepsilon-(\varepsilon-1) \gamma_{i}\right)^{p} \cdot\left(\varepsilon-(\varepsilon-1) \gamma_{j}\right)^{q}+(\varepsilon-1) \gamma_{i}^{p} \gamma_{j}^{q}}\right\},\right.\right. \\
& \bigcup_{\delta_{i} \in \widetilde{I}\left(a_{i}\right), \delta_{j} \in \widetilde{I}\left(a_{j}\right)}\left\{\frac{\left(1+(\varepsilon-1) \delta_{i}\right)^{p} \cdot\left(1+(\varepsilon-1) \delta_{j}\right)^{q}-\left(1-\delta_{i}\right)^{p}\left(1-\delta_{j}\right)^{q}}{\left(1+(\varepsilon-1) \delta_{i}\right)^{p} \cdot\left(1+(\varepsilon-1) \delta_{j}\right)^{q}+(\varepsilon-1)\left(1-\delta_{i}\right)^{p}\left(1-\delta_{j}\right)^{q}}\right\}, \\
& \left.\left.\bigcup_{\eta_{i} \in \widetilde{F}\left(a_{i}\right), \eta_{j} \in \widetilde{F}\left(a_{j}\right)}\left\{\frac{\left(1+(\varepsilon-1) \eta_{i}\right)^{p} \cdot\left(1+(\varepsilon-1) \eta_{j}\right)^{q}-\left(1-\eta_{i}\right)^{p}\left(1-\eta_{j}\right)^{q}}{\left(1+(\varepsilon-1) \eta_{i}\right)^{p} \cdot\left(1+(\varepsilon-1) \eta_{j}\right)^{q}+(\varepsilon-1)\left(1-\eta_{i}\right)^{p}\left(1-\eta_{j}\right)^{q}}\right\}\right)\right\rangle .
\end{aligned}
$$
below.

Firstly, we need to testify the mathematical formula

$$
\begin{aligned}
& \bigoplus_{\substack{i, j=1 \\
i \neq j}}^{n} \frac{\omega_{i} \omega_{j}}{1-\omega_{i}}\left(a_{i}^{p} \otimes a_{j}^{q}\right) \\
& \left(\prod _ { \substack { i , j = 1 \\
i \neq j } } ^ { n } \left(\left(\varepsilon-(\varepsilon-1) \gamma_{i}\right)^{p}\left(\varepsilon-(\varepsilon-1) \gamma_{j}\right)^{q}\right.\right. \\
& =\left\langle s_{\substack{i, j=1 \\
i \neq j}}^{n}\left(\omega_{i} \omega_{j} /\left(1-\omega_{i}\right)\right) \cdot \theta^{p}\left(a_{i}\right) \cdot \theta^{q}\left(a_{j}\right),\right. \\
& \left(\bigcup _ { \gamma _ { i } \in \widetilde { T } ( a _ { i } ) , \gamma _ { j } \in \widetilde { T } ( a _ { j } ) } \left\{\left(\prod _ { \substack { i , j = 1 \\
i \neq j } } ^ { n } \left(\left(\varepsilon-(\varepsilon-1) \gamma_{i}\right)^{p}\right.\right.\right.\right. \\
& \left.+\left(\varepsilon^{2}-1\right) \gamma_{i}^{p} \gamma_{j}^{q}\right)^{\omega_{i} \omega_{j} /\left(1-\omega_{i}\right)}+(\varepsilon-1) \\
& \cdot \prod_{\substack{i, j=1 \\
i \neq j}}^{n}\left(\left(\varepsilon-(\varepsilon-1) \gamma_{i}\right)^{p}\left(\varepsilon-(\varepsilon-1) \gamma_{j}\right)^{q}\right. \\
& \left.\left.\left.-\gamma_{i}^{p} \gamma_{j}^{q}\right)^{\omega_{i} \omega_{j} /\left(1-\omega_{i}\right)}\right)^{-1}\right\} \\
& \left.\cdot\left(\varepsilon-(\varepsilon-1) \gamma_{j}\right)^{q}+\left(\varepsilon^{2}-1\right) \gamma_{i}^{p} \gamma_{j}^{q}\right)^{\omega_{i} \omega_{j} /\left(1-\omega_{i}\right)} \\
& -\prod_{\substack{i, j=1 \\
i \neq j}}^{n}\left(\left(\varepsilon-(\varepsilon-1) \gamma_{i}\right)^{p}\right. \\
& \bigcup_{\delta_{i} \in \widetilde{I}\left(a_{i}\right), \delta_{j} \in \widetilde{I}\left(a_{j}\right)}\left\{\varepsilon \prod _ { \substack { i , j = 1 \\
i \neq j } } ^ { n } \left(\left(1+(\varepsilon-1) \delta_{i}\right)^{p}\left(1+(\varepsilon-1) \delta_{j}\right)^{q}\right.\right. \\
& \left.\left.\cdot\left(\varepsilon-(\varepsilon-1) \gamma_{j}\right)^{q}-\gamma_{i}^{p} \gamma_{j}^{q}\right)^{\omega_{i} \omega_{j} /\left(1-\omega_{i}\right)}\right) \\
& \left.-\left(1-\delta_{i}\right)^{p}\left(1-\delta_{j}\right)^{q}\right)^{\omega_{i} \omega_{j} /\left(1-\omega_{i}\right)} \\
& \left(\prod _ { \substack { i , j = 1 \\
i \neq j } } ^ { n } \left(\left(1+(\varepsilon-1) \delta_{i}\right)^{p}\left(1+(\varepsilon-1) \delta_{j}\right)^{q}\right.\right.
\end{aligned}
$$




$$
\begin{aligned}
& \left.+\left(\varepsilon^{2}-1\right)\left(1-\delta_{i}\right)^{p}\left(1-\delta_{j}\right)^{q}\right)^{\omega_{i} \omega_{j} /\left(1-\omega_{i}\right)} \\
& +(\varepsilon-1) \prod_{\substack{i, j=1 \\
i \neq j}}^{n}\left(\left(1+(\varepsilon-1) \delta_{i}\right)^{p}\left(1+(\varepsilon-1) \delta_{j}\right)^{q}\right. \\
& \left.\left.\left.-\left(1-\delta_{i}\right)^{p}\left(1-\delta_{j}\right)^{q}\right)^{\omega_{i} \omega_{j} /\left(1-\omega_{i}\right)}\right)^{-1}\right\} \text {, } \\
& \bigcup_{\eta_{i} \in \widetilde{F}\left(a_{i}\right), \eta_{j} \in \widetilde{F}\left(a_{j}\right)}\left\{\varepsilon \prod _ { \substack { i , j = 1 \\
i \neq j } } ^ { n } \left(\left(1+(\varepsilon-1) \eta_{i}\right)^{p}\left(1+(\varepsilon-1) \eta_{j}\right)^{q}\right.\right. \\
& \left.-\left(1-\eta_{i}\right)^{p}\left(1-\eta_{j}\right)^{q}\right)^{\omega_{i} \omega_{j} /\left(1-\omega_{i}\right)} \\
& \left(\prod _ { \substack { i , j = 1 \\
i \neq j } } ^ { n } \left(\left(1+(\varepsilon-1) \eta_{i}\right)^{p}\left(1+(\varepsilon-1) \eta_{j}\right)^{q}\right.\right. \\
& \left.+\left(\varepsilon^{2}-1\right)\left(1-\eta_{i}\right)^{p}\left(1-\eta_{j}\right)^{q}\right)^{\omega_{i} \omega_{j} /\left(1-\omega_{i}\right)} \\
& +(\varepsilon-1) \prod_{\substack{i, j=1 \\
i \neq j}}^{n}\left(\left(1+(\varepsilon-1) \eta_{i}\right)^{p}\left(1+(\varepsilon-1) \eta_{j}\right)^{q}\right.
\end{aligned}
$$

$\left.\left.\left.\left.\left.-\left(1-\eta_{i}\right)^{p}\left(1-\eta_{j}\right)^{q}\right)^{\omega_{i} \omega_{j} /\left(1-\omega_{i}\right)}\right)^{-1}\right\}\right)\right\rangle$

The mathematical induction on $n$ is adopted to prove (21). (1) Supposing $n=2$, the equation below is obtained.

$$
\begin{aligned}
& \bigoplus_{\substack{i, j=1 \\
i \neq j}}^{2} \frac{\omega_{i} \omega_{j}}{1-\omega_{i}}\left(a_{i}^{p} \otimes a_{j}^{q}\right)=\frac{\omega_{1} \omega_{2}}{1-\omega_{1}}\left(a_{1}^{p} \otimes a_{2}^{q}\right) \\
& \oplus \frac{\omega_{2} \omega_{1}}{1-\omega_{2}}\left(a_{2}^{p} \otimes a_{1}^{q}\right) \\
& =\left\langle s_{\left(\omega_{1} \omega_{2} /\left(1-\omega_{1}\right)\right) \cdot \theta^{p}\left(a_{1}\right) \cdot \theta^{q}\left(a_{2}\right)+\left(\omega_{2} \omega_{1} /\left(1-\omega_{2}\right)\right) \cdot \theta^{p}\left(a_{2}\right) \cdot \theta^{q}\left(a_{1}\right)},\right. \\
& \left(\bigcup_{\gamma_{i} \in \widetilde{T}\left(a_{i}\right), \gamma_{j} \in \widetilde{T}\left(a_{j}\right)}\left\{\frac{x_{1} x_{2}-y_{1} y_{2}}{x_{1} x_{2}+(\varepsilon-1) y_{1} y_{2}}\right\},\right. \\
& \bigcup_{\delta_{i} \in \tilde{I}\left(a_{i}\right), \delta_{j} \in \tilde{I}\left(a_{j}\right)}\left\{\frac{\varepsilon m_{1} m_{2}}{z_{1} z_{2}+(\varepsilon-1) m_{1} m_{2}}\right\}, \\
& \left.\left.\bigcup_{\eta_{i} \in \tilde{F}\left(a_{i}\right), \eta_{j} \in \widetilde{F}\left(a_{j}\right)}\left\{\frac{\varepsilon v_{1} v_{2}}{u_{1} u_{2}+(\varepsilon-1) v_{1} v_{2}}\right\}\right)\right\rangle
\end{aligned}
$$

$$
\begin{aligned}
& x_{1}=\left(1+(\varepsilon-1) \cdot \frac{\varepsilon \gamma_{1}^{p} \gamma_{2}{ }^{q}}{\left(\varepsilon-(\varepsilon-1) \gamma_{1}\right)^{p} \cdot\left(\varepsilon-(\varepsilon-1) \gamma_{2}\right)^{q}+(\varepsilon-1) \gamma_{1}{ }^{p} \gamma_{2}{ }^{q}}\right)^{\omega_{1} \omega_{2} /\left(1-\omega_{1}\right)}, \\
& x_{2}=\left(1+(\varepsilon-1) \cdot \frac{\varepsilon \gamma_{2}^{p} \gamma_{1}^{q}}{\left(\varepsilon-(\varepsilon-1) \gamma_{2}\right)^{p} \cdot\left(\varepsilon-(\varepsilon-1) \gamma_{1}\right)^{q}+(\varepsilon-1) \gamma_{2}^{p} \gamma_{1}^{q}}\right)^{\omega_{2} \omega_{1} /\left(1-\omega_{2}\right)} \text {, } \\
& y_{1}=\left(1-\frac{\varepsilon \gamma_{1}^{p} \gamma_{2}^{q}}{\left(\varepsilon-(\varepsilon-1) \gamma_{1}\right)^{p} \cdot\left(\varepsilon-(\varepsilon-1) \gamma_{2}\right)^{q}+(\varepsilon-1) \gamma_{1}^{p} \gamma_{2}^{q}}\right)^{\omega_{1} \omega_{2} /\left(1-\omega_{1}\right)} \text {, } \\
& y_{2}=\left(1-\frac{\varepsilon \gamma_{2}^{p} \gamma_{1}^{q}}{\left(\varepsilon-(\varepsilon-1) \gamma_{2}\right)^{p} \cdot\left(\varepsilon-(\varepsilon-1) \gamma_{1}\right)^{q}+(\varepsilon-1) \gamma_{2}^{p} \gamma_{1}^{q}}\right)^{\omega_{2} \omega_{1} /\left(1-\omega_{2}\right)} \text {, } \\
& m_{1}=\left(\frac{\left(1+(\varepsilon-1) \delta_{1}\right)^{p} \cdot\left(1+(\varepsilon-1) \delta_{2}\right)^{q}-\left(1-\delta_{1}\right)^{p}\left(1-\delta_{2}\right)^{q}}{\left(1+(\varepsilon-1) \delta_{1}\right)^{p} \cdot\left(1+(\varepsilon-1) \delta_{2}\right)^{q}+(\varepsilon-1)\left(1-\delta_{1}\right)^{p}\left(1-\delta_{2}\right)^{q}}\right)^{\omega_{1} \omega_{2} /\left(1-\omega_{1}\right)}, \\
& m_{2}=\left(\frac{\left(1+(\varepsilon-1) \delta_{2}\right)^{p} \cdot\left(1+(\varepsilon-1) \delta_{1}\right)^{q}-\left(1-\delta_{2}\right)^{p}\left(1-\delta_{1}\right)^{q}}{\left(1+(\varepsilon-1) \delta_{2}\right)^{p} \cdot\left(1+(\varepsilon-1) \delta_{1}\right)^{q}+(\varepsilon-1)\left(1-\delta_{2}\right)^{p}\left(1-\delta_{1}\right)^{q}}\right)^{\omega_{2} \omega_{1} /\left(1-\omega_{2}\right)} \\
& z_{1}=\left(1+(\varepsilon-1)\left(1-\frac{\left(1+(\varepsilon-1) \delta_{1}\right)^{p} \cdot\left(1+(\varepsilon-1) \delta_{2}\right)^{q}-\left(1-\delta_{1}\right)^{p}\left(1-\delta_{2}\right)^{q}}{\left(1+(\varepsilon-1) \delta_{1}\right)^{p} \cdot\left(1+(\varepsilon-1) \delta_{2}\right)^{q}+(\varepsilon-1)\left(1-\delta_{1}\right)^{p}\left(1-\delta_{2}\right)^{q}}\right)\right)^{\omega_{1} \omega_{2} /\left(1-\omega_{1}\right)}, \\
& z_{2}=\left(1+(\varepsilon-1)\left(1-\frac{\left(1+(\varepsilon-1) \delta_{2}\right)^{p} \cdot\left(1+(\varepsilon-1) \delta_{1}\right)^{q}-\left(1-\delta_{2}\right)^{p}\left(1-\delta_{1}\right)^{q}}{\left(1+(\varepsilon-1) \delta_{2}\right)^{p} \cdot\left(1+(\varepsilon-1) \delta_{1}\right)^{q}+(\varepsilon-1)\left(1-\delta_{2}\right)^{p}\left(1-\delta_{1}\right)^{q}}\right)\right)^{\omega_{2} \omega_{1} /\left(1-\omega_{2}\right)},
\end{aligned}
$$




$$
\begin{aligned}
& v_{1}=\left(\frac{\left(1+(\varepsilon-1) \eta_{1}\right)^{p} \cdot\left(1+(\varepsilon-1) \eta_{2}\right)^{q}-\left(1-\eta_{1}\right)^{p}\left(1-\eta_{2}\right)^{q}}{\left(1+(\varepsilon-1) \eta_{1}\right)^{p} \cdot\left(1+(\varepsilon-1) \eta_{2}\right)^{q}+(\varepsilon-1)\left(1-\eta_{1}\right)^{p}\left(1-\eta_{2}\right)^{q}}\right)^{\omega_{1} \omega_{2} /\left(1-\omega_{1}\right)}, \\
& v_{2}=\left(\frac{\left(1+(\varepsilon-1) \eta_{2}\right)^{p} \cdot\left(1+(\varepsilon-1) \eta_{1}\right)^{q}-\left(1-\eta_{2}\right)^{p}\left(1-\eta_{1}\right)^{q}}{\left(1+(\varepsilon-1) \eta_{2}\right)^{p} \cdot\left(1+(\varepsilon-1) \eta_{1}\right)^{q}+(\varepsilon-1)\left(1-\eta_{2}\right)^{p}\left(1-\eta_{1}\right)^{q}}\right)^{\omega_{2} \omega_{1} /\left(1-\omega_{2}\right)}, \\
& u_{1}=\left(1+(\varepsilon-1)\left(1-\frac{\left(1+(\varepsilon-1) \eta_{1}\right)^{p} \cdot\left(1+(\varepsilon-1) \eta_{2}\right)^{q}-\left(1-\eta_{1}\right)^{p}\left(1-\eta_{2}\right)^{q}}{\left(1+(\varepsilon-1) \eta_{1}\right)^{p} \cdot\left(1+(\varepsilon-1) \eta_{2}\right)^{q}+(\varepsilon-1)\left(1-\eta_{1}\right)^{p}\left(1-\eta_{2}\right)^{q}}\right)\right)^{\omega_{1} \omega_{2} /\left(1-\omega_{1}\right)}, \\
& u_{2}=\left(1+(\varepsilon-1)\left(1-\frac{\left(1+(\varepsilon-1) \eta_{2}\right)^{p} \cdot\left(1+(\varepsilon-1) \eta_{1}\right)^{q}-\left(1-\eta_{2}\right)^{p}\left(1-\eta_{1}\right)^{q}}{\left(1+(\varepsilon-1) \eta_{2}\right)^{p} \cdot\left(1+(\varepsilon-1) \eta_{1}\right)^{q}+(\varepsilon-1)\left(1-\eta_{2}\right)^{p}\left(1-\eta_{1}\right)^{q}}\right)\right)^{\omega_{2} \omega_{1} /\left(1-\omega_{2}\right)},
\end{aligned}
$$

and then,

$$
\begin{aligned}
& \bigoplus_{\substack{i, j=1 \\
i \neq j}}^{2} \frac{\omega_{i} \omega_{j}}{1-\omega_{i}}\left(a_{i}^{p} \otimes a_{j}^{q}\right) \\
& =\frac{\omega_{1} \omega_{2}}{1-\omega_{1}}\left(a_{1}^{p} \otimes a_{2}^{q}\right) \oplus \frac{\omega_{2} \omega_{1}}{1-\omega_{2}}\left(a_{2}^{p} \otimes a_{1}^{q}\right) \\
& =\left\langle s_{\substack{\sum_{i, j=1}^{2}\left(\left(\omega_{i} \omega_{j} /\left(1-\omega_{i}\right)\right) \cdot \theta^{p}\left(a_{i}\right) \cdot \theta^{q}\left(a_{j}\right)\right) \\
i \neq j}}\right. \\
& \left(\bigcup _ { \gamma _ { i } \in \widetilde { T } ( a _ { i } ) , \gamma _ { j } \in \tilde { T } ( a _ { j } ) } \left\{\left(\prod _ { \substack { i , j = 1 \\
i \neq j } } ^ { 2 } \left(\left(\varepsilon-(\varepsilon-1) \gamma_{i}\right)^{p}\left(\varepsilon-(\varepsilon-1) \gamma_{j}\right)^{q}\right.\right.\right.\right. \\
& \left.+\left(\varepsilon^{2}-1\right) \gamma_{i}^{p} \gamma_{j}^{q}\right)^{\omega_{i} \omega_{j} /\left(1-\omega_{i}\right)} \\
& -\prod_{\substack{i, j=1 \\
i \neq j}}^{2}\left(\left(\varepsilon-(\varepsilon-1) \gamma_{i}\right)^{p}\left(\varepsilon-(\varepsilon-1) \gamma_{j}\right)^{q}\right. \\
& \left.\left.-\gamma_{i}^{p} \gamma_{j}^{q}\right)^{\omega_{i} \omega_{j} /\left(1-\omega_{i}\right)}\right) \\
& \cdot\left(\prod _ { \substack { i , j = 1 \\
i \neq j } } ^ { 2 } \left(\left(\varepsilon-(\varepsilon-1) \gamma_{i}\right)^{p}\left(\varepsilon-(\varepsilon-1) \gamma_{j}\right)^{q}\right.\right. \\
& \left.+\left(\varepsilon^{2}-1\right) \gamma_{i}^{p} \gamma_{j}^{q}\right)^{\omega_{i} \omega_{j} /\left(1-\omega_{i}\right)} \\
& +(\varepsilon-1) \prod_{\substack{i, j=1 \\
i \neq j}}^{2}\left(\left(\varepsilon-(\varepsilon-1) \gamma_{i}\right)^{p}\left(\varepsilon-(\varepsilon-1) \gamma_{j}\right)^{q}\right. \\
& \left.\left.\left.-\gamma_{i}^{p} \gamma_{j}^{q}\right)^{\omega_{i} \omega_{j} /\left(1-\omega_{i}\right)}\right)^{-1}\right\}
\end{aligned}
$$

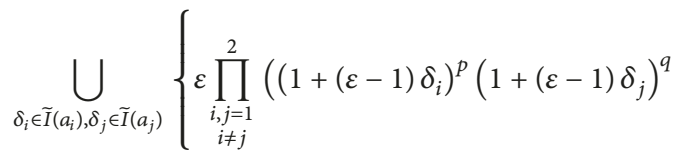

$$
\begin{aligned}
& \left.-\left(1-\delta_{i}\right)^{p}\left(1-\delta_{j}\right)^{q}\right)^{\omega_{i} \omega_{j} /\left(1-\omega_{i}\right)} \\
& \cdot\left(\prod _ { \substack { i , j = 1 \\
i \neq j } } ^ { 2 } \left(\left(1+(\varepsilon-1) \delta_{i}\right)^{p}\left(1+(\varepsilon-1) \delta_{j}\right)^{q}\right.\right. \\
& \left.+\left(\varepsilon^{2}-1\right)\left(1-\delta_{i}\right)^{p}\left(1-\delta_{j}\right)^{q}\right)^{\omega_{i} \omega_{j} /\left(1-\omega_{i}\right)} \\
& +(\varepsilon-1) \prod_{\substack{i, j=1 \\
i \neq j}}^{2}\left(\left(1+(\varepsilon-1) \delta_{i}\right)^{p}\left(1+(\varepsilon-1) \delta_{j}\right)^{q}\right. \\
& \left.\left.\left.-\left(1-\delta_{i}\right)^{p}\left(1-\delta_{j}\right)^{q}\right)^{\omega_{i} \omega_{j} /\left(1-\omega_{i}\right)}\right)^{-1}\right\} \text {, } \\
& \bigcup_{\eta_{i} \in \tilde{F}\left(a_{i}\right), \eta_{j} \in \tilde{F}\left(a_{j}\right)}\left\{\varepsilon \prod _ { \substack { i , j = 1 \\
i \neq j } } ^ { 2 } \left(\left(1+(\varepsilon-1) \eta_{i}\right)^{p}\left(1+(\varepsilon-1) \eta_{j}\right)^{q}\right.\right. \\
& \left.-\left(1-\eta_{i}\right)^{p}\left(1-\eta_{j}\right)^{q}\right)^{\omega_{i} \omega_{j} /\left(1-\omega_{i}\right)} \\
& \cdot\left(\prod _ { \substack { i , j = 1 \\
i \neq j } } ^ { 2 } \left(\left(1+(\varepsilon-1) \eta_{i}\right)^{p}\left(1+(\varepsilon-1) \eta_{j}\right)^{q}\right.\right. \\
& \left.+\left(\varepsilon^{2}-1\right)\left(1-\eta_{i}\right)^{p}\left(1-\eta_{j}\right)^{q}\right)^{\omega_{i} \omega_{j} /\left(1-\omega_{i}\right)} \\
& +(\varepsilon-1) \prod_{\substack{i, j=1 \\
i \neq j}}^{2}\left(\left(1+(\varepsilon-1) \eta_{i}\right)^{p}\left(1+(\varepsilon-1) \eta_{j}\right)^{q}\right. \\
& \left.\left.\left.\left.\left.-\left(1-\eta_{i}\right)^{p}\left(1-\eta_{j}\right)^{q}\right)^{\omega_{i} \omega_{j} /\left(1-\omega_{i}\right)}\right)^{-1}\right\}\right)\right\rangle .
\end{aligned}
$$

We can make (21) right when $n=k$. 
(2) Supposing $n=k$, the equation below is right; then

$$
\begin{aligned}
& \bigoplus_{\substack{i, j=1 \\
i \neq j}}^{k} \frac{\omega_{i} \omega_{j}}{1-\omega_{i}}\left(a_{i}^{p} \otimes a_{j}^{q}\right) \\
& =\left\langle s_{\substack{i, j=1 \\
i \neq j}}\left(\omega_{i} \omega_{j} /\left(1-\omega_{i}\right)\right) \cdot \theta^{p}\left(a_{i}\right) \cdot \theta^{q}\left(a_{j}\right),\right. \\
& \left(\bigcup _ { \gamma _ { i } \in \widetilde { T } ( a _ { i } ) , \gamma _ { j } \in \widetilde { T } ( a _ { j } ) } \left\{\left(\prod _ { \substack { i , j = 1 \\
i \neq j } } ^ { k } \left(\left(\varepsilon-(\varepsilon-1) \gamma_{i}\right)^{p}\right.\right.\right.\right. \\
& \left.\cdot\left(\varepsilon-(\varepsilon-1) \gamma_{j}\right)^{q}+\left(\varepsilon^{2}-1\right) \gamma_{i}^{p} \gamma_{j}^{q}\right)^{\omega_{i} \omega_{j} /\left(1-\omega_{i}\right)} \\
& -\prod_{\substack{i, j=1 \\
i \neq j}}^{k}\left(\left(\varepsilon-(\varepsilon-1) \gamma_{i}\right)^{p}\left(\varepsilon-(\varepsilon-1) \gamma_{j}\right)^{q}\right. \\
& \left.\left.-\gamma_{i}^{p} \gamma_{j}^{q}\right)^{\omega_{i} \omega_{j} /\left(1-\omega_{i}\right)}\right) \\
& \left(\prod _ { \substack { i , j = 1 \\
i \neq j } } ^ { k } \left(\left(\varepsilon-(\varepsilon-1) \gamma_{i}\right)^{p}\left(\varepsilon-(\varepsilon-1) \gamma_{j}\right)^{q}\right.\right. \\
& \left.+\left(\varepsilon^{2}-1\right) \gamma_{i}^{p} \gamma_{j}^{q}\right)^{\omega_{i} \omega_{j} /\left(1-\omega_{i}\right)} \\
& +(\varepsilon-1) \prod_{\substack{i, j=1 \\
i \neq j}}^{k}\left(\left(\varepsilon-(\varepsilon-1) \gamma_{i}\right)^{p}\left(\varepsilon-(\varepsilon-1) \gamma_{j}\right)^{q}\right. \\
& \left.\left.\left.-\gamma_{i}^{p} \gamma_{j}^{q}\right)^{\omega_{i} \omega_{j} /\left(1-\omega_{i}\right)}\right)^{-1}\right\} \text {, } \\
& \bigcup_{\delta_{i} \in \widetilde{I}\left(a_{i}\right), \delta_{j} \in \widetilde{I}\left(a_{j}\right)}\left\{\varepsilon \prod _ { \substack { i , j = 1 \\
i \neq j } } ^ { k } \left(\left(1+(\varepsilon-1) \delta_{i}\right)^{p}\right.\right. \\
& \cdot\left(1+(\varepsilon-1) \delta_{j}\right)^{q} \\
& \left.-\left(1-\delta_{i}\right)^{p}\left(1-\delta_{j}\right)^{q}\right)^{\omega_{i} \omega_{j} /\left(1-\omega_{i}\right)} \\
& \left(\prod _ { \substack { i , j = 1 \\
i \neq j } } ^ { k } \left(\left(1+(\varepsilon-1) \delta_{i}\right)^{p}\left(1+(\varepsilon-1) \delta_{j}\right)^{q}\right.\right. \\
& \left.+\left(\varepsilon^{2}-1\right)\left(1-\delta_{i}\right)^{p}\left(1-\delta_{j}\right)^{q}\right)^{\omega_{i} \omega_{j} /\left(1-\omega_{i}\right)}
\end{aligned}
$$

$$
\begin{aligned}
& +(\varepsilon-1) \prod_{\substack{i, j=1 \\
i \neq j}}^{k}\left(\left(1+(\varepsilon-1) \delta_{i}\right)^{p}\left(1+(\varepsilon-1) \delta_{j}\right)^{q}\right. \\
& \left.\left.\left.-\left(1-\delta_{i}\right)^{p}\left(1-\delta_{j}\right)^{q}\right)^{\omega_{i} \omega_{j} /\left(1-\omega_{i}\right)}\right)^{-1}\right\}, \\
& \bigcup_{\eta_{i} \in \tilde{F}\left(a_{i}\right), \eta_{j} \in \widetilde{F}\left(a_{j}\right)}\left\{\varepsilon \prod _ { \substack { i , j = 1 \\
i \neq j } } ^ { k } \left(\left(1+(\varepsilon-1) \eta_{i}\right)^{p}\right.\right. \\
& \left.\cdot\left(1+(\varepsilon-1) \eta_{j}\right)^{q}-\left(1-\eta_{i}\right)^{p}\left(1-\eta_{j}\right)^{q}\right)^{\omega_{i} \omega_{j} /\left(1-\omega_{i}\right)} \\
& \cdot\left(\prod _ { \substack { i , j = 1 \\
i \neq j } } ^ { k } \left(\left(1+(\varepsilon-1) \eta_{i}\right)^{p}\left(1+(\varepsilon-1) \eta_{j}\right)^{q}\right.\right. \\
& \left.+\left(\varepsilon^{2}-1\right)\left(1-\eta_{i}\right)^{p}\left(1-\eta_{j}\right)^{q}\right)^{\omega_{i} \omega_{j} /\left(1-\omega_{i}\right)} \\
& +(\varepsilon-1) \prod_{\substack{i, j=1 \\
i \neq j}}^{k}\left(\left(1+(\varepsilon-1) \eta_{i}\right)^{p}\left(1+(\varepsilon-1) \eta_{j}\right)^{q}\right. \\
& \left.\left.\left.\left.\left.-\left(1-\eta_{i}\right)^{p}\left(1-\eta_{j}\right)^{q}\right)^{\omega_{i} \omega_{j} /\left(1-\omega_{i}\right)}\right)^{-1}\right\}\right)\right\rangle .
\end{aligned}
$$

When $n=k+1$, we need to calculate the equation below.

$$
\begin{aligned}
& \bigoplus_{\substack{i, j=1 \\
i \neq j}}^{k+1} \frac{\omega_{i} \omega_{j}}{1-\omega_{i}}\left(a_{i}^{p} \otimes a_{j}^{q}\right) \\
& =\bigoplus_{\substack{i, j=1 \\
i \neq j}}^{k} \frac{\omega_{i} \omega_{j}}{1-\omega_{i}}\left(a_{i}^{p} \otimes a_{j}^{q}\right) \\
& \quad \oplus \bigoplus_{i=1}^{k} \frac{\omega_{i} \omega_{k+1}}{1-\omega_{i}}\left(a_{i}^{p} \otimes a_{k+1}^{q}\right) \\
& \quad \oplus \bigoplus_{j=1}^{k} \frac{\omega_{k+1} \omega_{j}}{1-\omega_{k+1}}\left(a_{k+1}^{p} \otimes a_{j}^{q}\right) .
\end{aligned}
$$

The mathematical induction on $k$ is used to testify the equations below.

$$
\begin{aligned}
& \bigoplus_{i=1}^{k} \frac{\omega_{i} \omega_{k+1}}{1-\omega_{i}}\left(a_{i}^{p} \otimes a_{k+1}^{q}\right) \\
& =\left\langle s_{\sum_{i=1}^{k}\left(\left(\omega_{i} \omega_{k+1} /\left(1-\omega_{i}\right)\right) \cdot \theta^{p}\left(a_{i}\right) \cdot \theta^{q}\left(a_{k+1}\right)\right)}\right.
\end{aligned}
$$


12

Mathematical Problems in Engineering

$$
\begin{aligned}
& \left(\bigcup _ { \gamma _ { i } \in \widetilde { T } ( a _ { i } ) , \gamma _ { k + 1 } \in \widetilde { T } ( a _ { k + 1 } ) } \left\{\left(\prod _ { i = 1 } ^ { k } \left(\left(\varepsilon-(\varepsilon-1) \gamma_{i}\right)^{p}\right.\right.\right.\right. \\
& \bigoplus_{j=1}^{k} \frac{\omega_{k+1} \omega_{j}}{1-\omega_{k+1}}\left(a_{k+1}^{p} \otimes a_{j}^{q}\right) \\
& \left.\cdot\left(\varepsilon-(\varepsilon-1) \gamma_{k+1}\right)^{q}+\left(\varepsilon^{2}-1\right) \gamma_{i}^{p} \gamma_{k+1}^{q}\right)^{\omega_{i} \omega_{k+1} /\left(1-\omega_{i}\right)} \\
& -\prod_{i=1}^{k}\left(\left(\varepsilon-(\varepsilon-1) \gamma_{i}\right)^{p}\left(\varepsilon-(\varepsilon-1) \gamma_{k+1}\right)^{q}\right. \\
& \left.\left.-\gamma_{i}^{p} \gamma_{k+1}^{q}\right)^{\omega_{i} \omega_{k+1} /\left(1-\omega_{i}\right)}\right) \\
& \left(\prod _ { i = 1 } ^ { k } \left(\left(\varepsilon-(\varepsilon-1) \gamma_{i}\right)^{p}\left(\varepsilon-(\varepsilon-1) \gamma_{k+1}\right)^{q}\right.\right. \\
& \left.+\left(\varepsilon^{2}-1\right) \gamma_{i}^{p} \gamma_{k+1}^{q}\right)^{\omega_{i} \omega_{k+1} /\left(1-\omega_{i}\right)} \\
& +(\varepsilon-1) \prod_{i=1}^{k}\left(\left(\varepsilon-(\varepsilon-1) \gamma_{i}\right)^{p}\left(\varepsilon-(\varepsilon-1) \gamma_{k+1}\right)^{q}\right. \\
& \left.\left.\left.-\gamma_{i}^{p} \gamma_{k+1}^{q}\right)^{\omega_{i} \omega_{k+1} /\left(1-\omega_{i}\right)}\right)^{-1}\right\} \\
& =\left\langle s_{\sum_{j=1}^{k}\left(\left(\omega_{k+1} \omega_{j} /\left(1-\omega_{k+1}\right)\right) \cdot \theta^{p}\left(a_{k+1}\right) \cdot \theta^{q}\left(a_{j}\right)\right)}\right. \\
& \left(\bigcup _ { \gamma _ { k + 1 } \in \widetilde { T } ( a _ { k + 1 } ) , \gamma _ { j } \in \widetilde { T } ( a _ { j } ) } \left\{\left(\prod _ { j = 1 } ^ { k } \left(\left(\varepsilon-(\varepsilon-1) \gamma_{k+1}\right)^{p}\right.\right.\right.\right. \\
& \left.\cdot\left(\varepsilon-(\varepsilon-1) \gamma_{j}\right)^{q}+\left(\varepsilon^{2}-1\right) \gamma_{k+1}{ }^{p} \gamma_{j}^{q}\right)^{\omega_{k+1} \omega_{j} /\left(1-\omega_{k+1}\right)} \\
& -\prod_{j=1}^{k}\left(\left(\varepsilon-(\varepsilon-1) \gamma_{k+1}\right)^{p}\left(\varepsilon-(\varepsilon-1) \gamma_{j}\right)^{q}\right. \\
& \left.\left.-\gamma_{k+1} p \gamma_{j}^{q}\right)^{\omega_{k+1} \omega_{j} /\left(1-\omega_{k+1}\right)}\right) \\
& \cdot\left(\prod _ { j = 1 } ^ { k } \left(\left(\varepsilon-(\varepsilon-1) \gamma_{k+1}\right)^{p}\left(\varepsilon-(\varepsilon-1) \gamma_{j}\right)^{q}\right.\right. \\
& \left.+\left(\varepsilon^{2}-1\right) \gamma_{k+1}{ }^{p} \gamma_{j}^{q}\right)^{\omega_{k+1} \omega_{j} /\left(1-\omega_{k+1}\right)} \\
& +(\varepsilon-1) \prod_{j=1}^{k}\left(\left(\varepsilon-(\varepsilon-1) \gamma_{k+1}\right)^{p}\left(\varepsilon-(\varepsilon-1) \gamma_{j}\right)^{q}\right. \\
& \left.\left.\left.-\gamma_{k+1} p \gamma_{j}^{q}\right)^{\omega_{k+1} \omega_{j} /\left(1-\omega_{k+1}\right)}\right)^{-1}\right\} \text {, } \\
& \left.+\left(\varepsilon^{2}-1\right)\left(1-\delta_{i}\right)^{p}\left(1-\delta_{k+1}\right)^{q}\right)^{\omega_{i} \omega_{k+1} /\left(1-\omega_{i}\right)} \\
& +(\varepsilon-1) \prod_{i=1}^{k}\left(\left(1+(\varepsilon-1) \delta_{i}\right)^{p}\left(1+(\varepsilon-1) \delta_{k+1}\right)^{q}\right. \\
& \left.\left.\left.-\left(1-\delta_{i}\right)^{p}\left(1-\delta_{k+1}\right)^{q}\right)^{\omega_{i} \omega_{k+1} /\left(1-\omega_{i}\right)}\right)^{-1}\right\} \text {, } \\
& \bigcup_{\delta_{k+1} \in \tilde{I}\left(a_{k+1}\right), \delta_{j} \in \tilde{I}\left(a_{j}\right)}\left\{\varepsilon \prod _ { j = 1 } ^ { k } \left(\left(1+(\varepsilon-1) \delta_{k+1}\right)^{p}\right.\right. \\
& \cdot\left(1+(\varepsilon-1) \delta_{j}\right)^{q} \\
& \left.-\left(1-\delta_{k+1}\right)^{p}\left(1-\delta_{j}\right)^{q}\right)^{\omega_{k+1} \omega_{j} /\left(1-\omega_{k+1}\right)} \\
& \left(\prod _ { j = 1 } ^ { k } \left(\left(1+(\varepsilon-1) \delta_{k+1}\right)^{p}\left(1+(\varepsilon-1) \delta_{j}\right)^{q}\right.\right. \\
& \left.+\left(\varepsilon^{2}-1\right)\left(1-\delta_{k+1}\right)^{p}\left(1-\delta_{j}\right)^{q}\right)^{\omega_{k+1} \omega_{j} /\left(1-\omega_{k+1}\right)} \\
& +(\varepsilon-1) \prod_{j=1}^{k}\left(\left(1+(\varepsilon-1) \delta_{k+1}\right)^{p}\left(1+(\varepsilon-1) \delta_{j}\right)^{q}\right. \\
& \left.\left.\left.-\left(1-\delta_{k+1}\right)^{p}\left(1-\delta_{j}\right)^{q}\right)^{\omega_{k+1} \omega_{j} /\left(1-\omega_{k+1}\right)}\right)^{-1}\right\} \text {, } \\
& +(\varepsilon-1) \prod_{i=1}^{k}\left(\left(1+(\varepsilon-1) \eta_{i}\right)^{p}\left(1+(\varepsilon-1) \eta_{k+1}\right)^{q}\right. \\
& \left.\left.\left.\left.\left.-\left(1-\eta_{i}\right)^{p}\left(1-\eta_{k+1}\right)^{q}\right)^{\omega_{i} \omega_{k+1} /\left(1-\omega_{i}\right)}\right)^{-1}\right\}\right)\right\rangle \text {, } \\
& \bigcup_{\eta_{k+1} \in \widetilde{F}\left(a_{k+1}\right), \eta_{j} \in \widetilde{F}\left(a_{j}\right)}\left\{\varepsilon \prod _ { j = 1 } ^ { k } \left(\left(1+(\varepsilon-1) \eta_{k+1}\right)^{p}\right.\right. \\
& \cdot\left(1+(\varepsilon-1) \eta_{j}\right)^{q}
\end{aligned}
$$


$\left.-\left(1-\eta_{k+1}\right)^{p}\left(1-\eta_{j}\right)^{q}\right)^{\omega_{k+1} \omega_{j} /\left(1-\omega_{k+1}\right)}$

$$
\begin{aligned}
& \cdot\left(\prod _ { j = 1 } ^ { k } \left(\left(1+(\varepsilon-1) \eta_{k+1}\right)^{p}\left(1+(\varepsilon-1) \eta_{j}\right)^{q}\right.\right. \\
& \left.+\left(\varepsilon^{2}-1\right)\left(1-\eta_{k+1}\right)^{p}\left(1-\eta_{j}\right)^{q}\right)^{\omega_{k+1} \omega_{j} /\left(1-\omega_{k+1}\right)} \\
& +(\varepsilon-1) \prod_{j=1}^{k}\left(\left(1+(\varepsilon-1) \eta_{k+1}\right)^{p}\left(1+(\varepsilon-1) \eta_{j}\right)^{q}\right. \\
& \left.\left.\left.\left.\left.-\left(1-\eta_{k+1}\right)^{p}\left(1-\eta_{j}\right)^{q}\right)^{\omega_{k+1} \omega_{j} /\left(1-\omega_{k+1}\right)}\right)^{-1}\right\}\right)\right\rangle .
\end{aligned}
$$

Therefore,

$$
\begin{aligned}
& \bigoplus_{\substack{i, j=1 \\
i \neq j}}^{k+1} \frac{\omega_{i} \omega_{j}}{1-\omega_{i}}\left(a_{i}^{p} \otimes a_{j}^{q}\right) \\
& =\bigoplus_{\substack{i, j=1 \\
i \neq j}}^{k} \frac{\omega_{i} \omega_{j}}{1-\omega_{i}}\left(a_{i}^{p} \otimes a_{j}^{q}\right) \\
& \oplus \bigoplus_{i=1}^{k} \frac{\omega_{i} \omega_{k+1}}{1-\omega_{i}}\left(a_{i}^{p} \otimes a_{k+1}^{q}\right) \\
& \oplus \bigoplus_{j=1}^{k} \frac{\omega_{k+1} \omega_{j}}{1-\omega_{k+1}}\left(a_{k+1}^{p} \otimes a_{j}^{q}\right)
\end{aligned}
$$

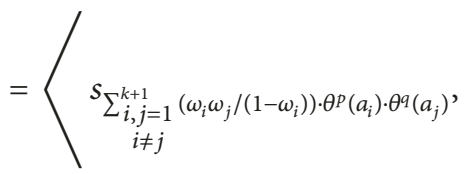

$$
\begin{aligned}
& \left(\bigcup _ { \gamma _ { i } \in \widetilde { T } ( a _ { i } ) , \gamma _ { j } \in \widetilde { T } ( a _ { j } ) } \left\{\left(\prod _ { \substack { i , j = 1 \\
i \neq j } } ^ { k + 1 } \left(\left(\varepsilon-(\varepsilon-1) \gamma_{i}\right)^{p}\right.\right.\right.\right. \\
& \left.\cdot\left(\varepsilon-(\varepsilon-1) \gamma_{j}\right)^{q}+\left(\varepsilon^{2}-1\right) \gamma_{i}^{p} \gamma_{j}^{q}\right)^{\omega_{i} \omega_{j} /\left(1-\omega_{i}\right)} \\
& -\prod_{\substack{i, j=1 \\
i \neq j}}^{k+1}\left(\left(\varepsilon-(\varepsilon-1) \gamma_{i}\right)^{p}\left(\varepsilon-(\varepsilon-1) \gamma_{j}\right)^{q}\right. \\
& \left.\left.-\gamma_{i}^{p} \gamma_{j}^{q}\right)^{\omega_{i} \omega_{j} /\left(1-\omega_{i}\right)}\right) \\
& \left(\prod _ { \substack { i , j = 1 \\
i \neq j } } ^ { k + 1 } \left(\left(\varepsilon-(\varepsilon-1) \gamma_{i}\right)^{p}\left(\varepsilon-(\varepsilon-1) \gamma_{j}\right)^{q}\right.\right.
\end{aligned}
$$

$$
\begin{aligned}
& \left.+\left(\varepsilon^{2}-1\right) \gamma_{i}^{p} \gamma_{j}^{q}\right)^{\omega_{i} \omega_{j} /\left(1-\omega_{i}\right)} \\
& +(\varepsilon-1) \prod_{\substack{i, j=1 \\
i \neq j}}^{k+1}\left(\left(\varepsilon-(\varepsilon-1) \gamma_{i}\right)^{p}\left(\varepsilon-(\varepsilon-1) \gamma_{j}\right)^{q}\right. \\
& \left.\left.\left.-\gamma_{i}^{p} \gamma_{j}^{q}\right)^{\omega_{i} \omega_{j} /\left(1-\omega_{i}\right)}\right)^{-1}\right\} \\
& \bigcup_{\delta_{i} \in \widetilde{I}\left(a_{i}\right), \delta_{j} \in \widetilde{I}\left(a_{j}\right)}\left\{\varepsilon \prod _ { \substack { i , j = 1 \\
i \neq j } } ^ { k + 1 } \left(\left(1+(\varepsilon-1) \delta_{i}\right)^{p}\right.\right. \\
& \cdot\left(1+(\varepsilon-1) \delta_{j}\right)^{q} \\
& \left.-\left(1-\delta_{i}\right)^{p}\left(1-\delta_{j}\right)^{q}\right)^{\omega_{i} \omega_{j} /\left(1-\omega_{i}\right)} \\
& \cdot\left(\prod _ { \substack { i , j = 1 \\
i \neq j } } ^ { k + 1 } \left(\left(1+(\varepsilon-1) \delta_{i}\right)^{p}\left(1+(\varepsilon-1) \delta_{j}\right)^{q}\right.\right. \\
& \left.+\left(\varepsilon^{2}-1\right)\left(1-\delta_{i}\right)^{p}\left(1-\delta_{j}\right)^{q}\right)^{\omega_{i} \omega_{j} /\left(1-\omega_{i}\right)} \\
& +(\varepsilon-1) \prod_{\substack{i, j=1 \\
i \neq j}}^{k+1}\left(\left(1+(\varepsilon-1) \delta_{i}\right)^{p}\left(1+(\varepsilon-1) \delta_{j}\right)^{q}\right. \\
& \left.\left.\left.-\left(1-\delta_{i}\right)^{p}\left(1-\delta_{j}\right)^{q}\right)^{\omega_{i} \omega_{j} /\left(1-\omega_{i}\right)}\right)^{-1}\right\} \text {, } \\
& \bigcup_{\eta_{i} \in \widetilde{F}\left(a_{i}\right), \eta_{j} \in \widetilde{F}\left(a_{j}\right)}\left\{\varepsilon \prod _ { \substack { i , j = 1 \\
i \neq j } } ^ { k + 1 } \left(\left(1+(\varepsilon-1) \eta_{i}\right)^{p}\right.\right. \\
& \left.\cdot\left(1+(\varepsilon-1) \eta_{j}\right)^{q}-\left(1-\eta_{i}\right)^{p}\left(1-\eta_{j}\right)^{q}\right)^{\omega_{i} \omega_{j} /\left(1-\omega_{i}\right)} \\
& \cdot\left(\prod _ { \substack { i , j = 1 \\
i \neq j } } ^ { k + 1 } \left(\left(1+(\varepsilon-1) \eta_{i}\right)^{p}\left(1+(\varepsilon-1) \eta_{j}\right)^{q}\right.\right. \\
& \left.+\left(\varepsilon^{2}-1\right)\left(1-\eta_{i}\right)^{p}\left(1-\eta_{j}\right)^{q}\right)^{\omega_{i} \omega_{j} /\left(1-\omega_{i}\right)} \\
& +(\varepsilon-1) \prod_{\substack{i, j=1 \\
i \neq j}}^{k+1}\left(\left(1+(\varepsilon-1) \eta_{i}\right)^{p}\left(1+(\varepsilon-1) \eta_{j}\right)^{q}\right. \\
& \left.\left.\left.\left.\left.-\left(1-\eta_{i}\right)^{p}\left(1-\eta_{j}\right)^{q}\right)^{\omega_{i} \omega_{j} /\left(1-\omega_{i}\right)}\right)^{-1}\right\}\right)\right\rangle .
\end{aligned}
$$


That is, If $n=k+1,(21)$ is right. Therefore, for all $n,(21)$ is right.

Then, (18) is right.

In the following, the properties of MVNLNWBMH operator will be proved.

(1) Reducibility. Let $\omega=(1 / n, 1 / n, \ldots, 1 / n)$. Then $\operatorname{MVNLNWBMH}\left(a_{1}, a_{2}, \ldots, a_{n}\right)=\operatorname{MVNLBMH}\left(a_{1}, a_{2}, \ldots\right.$, $\left.a_{n}\right)$.

Proof. Since $\omega=(1 / n, 1 / n, \ldots, 1 / n)$, then according to the operations in Definition 11, the result below can be obtained.

$$
\begin{aligned}
& \operatorname{MVNLNWBMH}\left(a_{1}, a_{2}, \ldots, a_{n}\right) \\
& =\left(\bigoplus_{\substack{i, j=1 \\
i \neq j}}^{n} \frac{\omega_{i} \omega_{j}}{1-\omega_{i}}\left(a_{i}^{p} \otimes a_{j}^{q}\right)\right)^{1 /(p+q)} \\
& =\left(\bigoplus_{\substack{i, j=1 \\
i \neq j}}^{n} \frac{1 / n^{2}}{1-1 / n}\left(a_{i}^{p} \otimes a_{j}^{q}\right)\right)^{1 /(p+q)} \\
& =\left(\bigoplus_{\substack{i, j=1 \\
i \neq j}}^{n} \frac{1}{n(n-1)}\left(a_{i}^{p} \otimes a_{j}^{q}\right)\right)^{1 /(p+q)} \\
& =\left(\frac{1}{n(n-1)} \bigoplus_{\substack{i, j=1 \\
i \neq j}}^{n}\left(a_{i}^{p} \otimes a_{j}^{q}\right)\right)^{1 /(p+q)} \\
& =\operatorname{MVNLBMH}\left(a_{1}, a_{2}, \ldots, a_{n}\right) \text {. }
\end{aligned}
$$

(2) Idempotency. Let $a_{i}=a(i=1,2, \ldots, n)$. Then $\operatorname{MVNLNWBMH}\left(a_{1}, a_{2}, \ldots, a_{n}\right)=a$.

Proof. For each $i$, owing to $a_{i}=a$, the formula below is obtained on the basis of (5) in Theorem 8 .

$$
\begin{aligned}
& \operatorname{MVNLNWBMH}\left(a_{1}, a_{2}, \ldots, a_{n}\right) \\
& =\left(\bigoplus_{\substack{i, j=1 \\
i \neq j}}^{n} \frac{\omega_{i} \omega_{j}}{1-\omega_{i}}\left(a_{i}^{p} \otimes a_{j}^{q}\right)\right)^{1 /(p+q)} \\
& =\left(\bigoplus_{\substack{i, j=1 \\
i \neq j}}^{n} \frac{\omega_{i} \omega_{j}}{1-\omega_{i}}\left(a^{p} \otimes a^{q}\right)\right)^{1 /(p+q)}
\end{aligned}
$$

$$
\begin{aligned}
& =\left(\bigoplus_{\substack{i, j=1 \\
i \neq j}}^{n} \frac{\omega_{i} \omega_{j}}{1-\omega_{i}} a^{p+q}\right)^{1 /(p+q)} \\
& =a\left(\bigoplus_{\substack{i, j=1 \\
i \neq j}}^{n} \frac{\omega_{i} \omega_{j}}{1-\omega_{i}}\right)^{1 /(p+q)}=a .
\end{aligned}
$$

(3) Commutativity. Let $\left(\widetilde{a}_{1}, \tilde{a}_{2}, \ldots, \widetilde{a}_{n}\right)$ be any permutation of $\left(a_{1}, a_{2}, \ldots, a_{n}\right)$. Then

$$
\begin{aligned}
& \operatorname{MVNLNWBMH}\left(\tilde{a}_{1}, \tilde{a}_{2}, \ldots, \tilde{a}_{n}\right) \\
& \quad=\operatorname{MVNLNWBMH}\left(a_{1}, a_{2}, \ldots, a_{n}\right) .
\end{aligned}
$$

Proof. As $\left(\tilde{a}_{1}, \tilde{a}_{2}, \ldots, \tilde{a}_{n}\right)$ is permutation of $\left(a_{1}, a_{2}, \ldots, a_{n}\right)$, then the equation below can be obtained.

$$
\begin{aligned}
& \operatorname{MVNLNWBMH}\left(a_{1}, a_{2}, \ldots, a_{n}\right) \\
& =\left(\bigoplus_{\substack{i, j=1 \\
i \neq j}}^{n} \frac{\omega_{i} \omega_{j}}{1-\omega_{i}}\left(a_{i}^{p} \otimes a_{j}^{q}\right)\right)^{1 /(p+q)} \\
& =\left(\bigoplus_{\substack{i, j=1 \\
i \neq j}}^{n} \frac{\omega_{i} \omega_{j}}{1-\omega_{i}}\left(\tilde{a}_{i}^{p} \otimes \tilde{a}_{j}^{q}\right)\right)^{1 /(p+q)} .
\end{aligned}
$$

(4) Monotonicity. Suppose $a_{i}=\left\langle s_{\theta\left(a_{i}\right)},\left(\widetilde{T}\left(a_{i}\right), \widetilde{I}\left(a_{i}\right), \widetilde{F}\left(a_{i}\right)\right)\right\rangle$ $(i=1,2, \ldots, n)$ and $b_{i}=\left\langle s_{\theta\left(b_{i}\right)},\left(\widetilde{T}\left(b_{i}\right), \widetilde{I}\left(b_{i}\right), \widetilde{F}\left(b_{i}\right)\right)\right\rangle(i=1,2$, $\ldots, n)$ are two sets of MVNLNs; when $s_{\theta\left(a_{i}\right)} \geq s_{\theta\left(b_{i}\right)}, \widetilde{T}\left(a_{i}\right) \geq$ $\widetilde{T}\left(b_{i}\right), \widetilde{I}\left(a_{i}\right) \leq \widetilde{I}\left(b_{i}\right)$, and $\widetilde{F}\left(a_{i}\right) \leq \widetilde{F}\left(b_{i}\right)$ for each $i$, then $\operatorname{MVNLNWBMH}\left(a_{1}, a_{2}, \ldots, a_{n}\right) \geq \operatorname{MVNLNWBMH}\left(b_{1}, b_{2}\right.$, $\left.\ldots, b_{n}\right)$.

Proof.

(I) Linguistic Term Part. As $p, q \geq 0$, and $s_{\theta\left(a_{i}\right)} \geq s_{\theta\left(b_{i}\right)}$ for each $i$, the result below is gained.

$$
\begin{aligned}
& \theta^{p}\left(a_{i}\right) \geq \theta^{p}\left(b_{i}\right), \\
& \theta^{q}\left(a_{i}\right) \geq \theta^{q}\left(b_{i}\right) \\
& \Downarrow \\
& \theta^{p}\left(a_{i}\right) \theta^{q}\left(a_{i}\right) \geq \theta^{p}\left(b_{i}\right) \theta^{q}\left(b_{i}\right) \\
& \Downarrow \\
& \frac{\omega_{i} \omega_{j}}{1-\omega_{i}} \theta^{p}\left(a_{i}\right) \theta^{q}\left(a_{i}\right) \geq \frac{\omega_{i} \omega_{j}}{1-\omega_{i}} \theta^{p}\left(b_{i}\right) \theta^{q}\left(b_{i}\right)
\end{aligned}
$$




$$
\begin{aligned}
& \sum_{\substack{i, j=1 \\
i \neq j}}^{n} \frac{\omega_{i} \omega_{j}}{1-\omega_{i}} \theta^{p}\left(a_{i}\right) \theta^{q}\left(a_{i}\right) \geq \sum_{\substack{i, j=1 \\
i \neq j}}^{n} \frac{\omega_{i} \omega_{j}}{1-\omega_{i}} \theta^{p}\left(b_{i}\right) \theta^{q}\left(b_{i}\right) \\
& \Downarrow \\
& \left(\sum_{\substack{i, j=1 \\
i \neq j}}^{n} \frac{\omega_{i} \omega_{j}}{1-\omega_{i}} \theta^{p}\left(a_{i}\right) \theta^{q}\left(a_{i}\right)\right)^{1 /(p+q)} \\
& \geq\left(\sum_{\substack{i, j=1 \\
i \neq j}}^{n} \frac{\omega_{i} \omega_{j}}{1-\omega_{i}} \theta^{p}\left(b_{i}\right) \theta^{q}\left(b_{i}\right)\right)^{1 /(p+q)} .
\end{aligned}
$$

(II) True-, Indeterminacy-, and Falsity-Membership Parts. As $\widetilde{T}\left(a_{i}\right) \geq \widetilde{T}\left(b_{i}\right), \widetilde{I}\left(a_{i}\right) \leq \widetilde{I}\left(b_{i}\right)$, and $\widetilde{F}\left(a_{i}\right) \leq \widetilde{F}\left(b_{i}\right)$ for each $i, p, q \geq$ 0 , then the following results can be proved easily.

$$
\begin{aligned}
& \frac{\varepsilon\left(x_{a_{i}}-y_{a_{i}}\right)^{1 /(p+q)}}{\left(x_{a_{i}}+\left(\varepsilon^{2}-1\right) y_{a_{i}}\right)^{1 /(p+q)}+(\varepsilon-1)\left(x_{a_{i}}-y_{a_{i}}\right)^{1 /(p+q)}} \\
& \geq \frac{\varepsilon\left(x_{b_{i}}-y_{b_{i}}\right)^{1 /(p+q)}}{\left(x_{b_{i}}+\left(\varepsilon^{2}-1\right) y_{b_{i}}\right)^{1 /(p+q)}+(\varepsilon-1)\left(x_{b_{i}}-y_{b_{i}}\right)^{1 /(p+q)}} \\
& \frac{\left(g_{a_{i}}+\left(\varepsilon^{2}-1\right) h_{a_{i}}\right)^{1 /(p+q)}-\left(g_{a_{i}}-h_{a_{i}}\right)^{1 /(p+q)}}{\left(g_{a_{i}}+\left(\varepsilon^{2}-1\right) h_{a_{i}}\right)^{1 /(p+q)}+(\varepsilon-1)\left(g_{a_{i}}-h_{a_{i}}\right)^{1 /(p+q)}} \\
& \leq \frac{\left(g_{b_{i}}+\left(\varepsilon^{2}-1\right) h_{b_{i}}\right)^{1 /(p+q)}-\left(g_{b_{i}}-h_{b_{i}}\right)^{1 /(p+q)}}{\left(g_{b_{i}}+\left(\varepsilon^{2}-1\right) h_{b_{i}}\right)^{1 /(p+q)}+(\varepsilon-1)\left(g_{b_{i}}-h_{b_{i}}\right)^{1 /(p+q)}} \\
& \frac{\left(v_{a_{i}}+\left(\varepsilon^{2}-1\right) u_{a_{i}}\right)^{1 /(p+q)}-\left(v_{a_{i}}-u_{a_{i}}\right)^{1 /(p+q)}}{\left(v_{a_{i}}+\left(\varepsilon^{2}-1\right) u_{a_{i}}\right)^{1 /(p+q)}+(\varepsilon-1)\left(v_{a_{i}}-u_{a_{i}}\right)^{1 /(p+q)}} \\
& \leq \frac{\left(v_{b_{i}}+\left(\varepsilon^{2}-1\right) u_{b_{i}}\right)^{1 /(p+q)}-\left(v_{b_{i}}-u_{b_{i}}\right)^{1 /(p+q)}}{\left(v_{b_{i}}+\left(\varepsilon^{2}-1\right) u_{b_{i}}\right)^{1 /(p+q)}+(\varepsilon-1)\left(v_{b_{i}}-u_{b_{i}}\right)^{1 /(p+q)}}
\end{aligned}
$$

where the corresponding $x, y, g, h, u$, and $v$ are defined in Definition 11.

(III) Comparing MVNLNWBMH $\left(a_{1}, a_{2}, \ldots, a_{n}\right)$ with $\operatorname{MVNLNWBMH}\left(b_{1}, b_{2}, \ldots, b_{n}\right)$. Suppose $a=\left\langle s_{\theta(a)},(\widetilde{T}(a)\right.$, $\widetilde{I}(a), \widetilde{F}(a))\rangle=\operatorname{MVNLNWBMH}\left(a_{1}, a_{2}, \ldots, a_{n}\right)$ and $b=$ $\left\langle s_{\theta(b)},(\widetilde{T}(b), \widetilde{I}(b), \widetilde{F}(b))\right\rangle=\operatorname{MVNLNWBMH}\left(b_{1}, b_{2}, \ldots, b_{n}\right)$.

Because $s_{\theta(a)} \geq s_{\theta(b)}, \widetilde{T}(a) \geq \widetilde{T}(b), \widetilde{I}(a) \leq \widetilde{I}(b)$, and $\widetilde{F}(a) \leq$ $\widetilde{F}(b)$, then $a \geq b$.

Then MVNLNWBMH $\left(a_{1}, a_{2}, \ldots, a_{n}\right) \geq$ MVNLNWB$\operatorname{MH}\left(b_{1}, b_{2}, \ldots, b_{n}\right)$.
In the following, a few special examples of MVNLNWBMH operator regarding different values $\varepsilon, p$, and $q$ will be explored.

(1) If $q=0$, then the MVNLNWBMH operator defined by (18) will be reduced to the generalized multivalued neutrosophic linguistic Hamacher weighted average (GMVNLHWA) operator shown as below.

$$
\begin{aligned}
& \operatorname{GMVNLHWA}\left(a_{1}, a_{2}, \ldots, a_{n}\right) \\
& =\left(\bigoplus_{\substack{i, j=1 \\
i \neq j}}^{n} \frac{\omega_{i} \omega_{j}}{1-\omega_{i}}\left(a_{i}^{p} \otimes a_{j}^{q}\right)\right)^{1 /(p+q)}=\left(\bigoplus_{\substack{i, j=1 \\
i \neq j}}^{n} \frac{\omega_{i} \omega_{j}}{1-\omega_{i}} a_{i}^{p}\right)^{1 / p} \\
& =\left(\bigoplus_{i=1}^{n} \frac{\omega_{i}\left(1-\omega_{i}\right)}{1-\omega_{i}} a_{i}^{p}\right)^{1 / p}=\left(\bigoplus_{i=1}^{n} \omega_{i} a_{i}^{p}\right)^{1 / p} \\
& =\left\langle s_{\left(\sum_{i=1}^{n}\left(\omega_{i} \cdot \theta^{p}\left(a_{i}\right)\right)\right)^{1 / p}},\right. \\
& \left(\underset{p_{i} \in \tilde{T}\left(a_{i}\right), y_{j} \in \tilde{T}\left(a_{j}\right)}{U}\left\{\frac{\varepsilon\left(x^{\prime}-y^{\prime}\right)^{1 / p}}{\left(x^{\prime}+\left(\varepsilon^{2}-1\right) y^{\prime}\right)^{1 / p}+(\varepsilon-1)\left(x^{\prime}-y^{\prime}\right)^{1 / p}}\right\},\right. \\
& \delta_{\delta_{i} \in\left(a_{i}\right), \delta_{j} \in \tilde{I}\left(a_{j}\right)}\left\{\frac{\left(g^{\prime}+\left(\varepsilon^{2}-1\right) h^{\prime}\right)^{1 / p}-\left(g^{\prime}-h^{\prime}\right)^{1 / p}}{\left(g^{\prime}+\left(\varepsilon^{2}-1\right) h^{\prime}\right)^{1 / p}+(\varepsilon-1)\left(g^{\prime}-h^{\prime}\right)^{1 / p}}\right\}, \\
& \left.\left.\underset{\eta_{i} \tilde{F}\left(a_{i}\right), \eta_{j} \in \tilde{F}\left(a_{j}\right)}{ }\left\{\frac{\left(v^{\prime}+\left(\varepsilon^{2}-1\right) u^{\prime}\right)^{1 / p}-\left(v^{\prime}-u^{\prime}\right)^{1 / p}}{\left(v^{\prime}+\left(\varepsilon^{2}-1\right) u^{\prime}\right)^{1 / p}+(\varepsilon-1)\left(v^{\prime}-u^{\prime}\right)^{1 / p}}\right\}\right)\right\rangle,
\end{aligned}
$$

where

$$
\begin{aligned}
& x^{\prime}=\prod_{i=1}^{n}\left(\left(1+(\varepsilon-1)\left(1-\gamma_{i}\right)\right)^{p}+\left(\varepsilon^{2}-1\right) \gamma_{i}^{p}\right)^{\omega_{i}}, \\
& y^{\prime}=\prod_{i=1}^{n}\left(\left(\varepsilon-(\varepsilon-1) \gamma_{i}\right)^{p}-\gamma_{i}^{p}\right)^{\omega_{i}}, \\
& h^{\prime}=\prod_{i=1}^{n}\left(\left(1+(\varepsilon-1) \delta_{i}\right)^{p}-\left(1-\delta_{i}\right)^{p}\right)^{\omega_{i}}, \\
& g^{\prime}=\prod_{i=1}^{n}\left(\left(1+(\varepsilon-1) \delta_{i}\right)^{p}+\left(\varepsilon^{2}-1\right)\left(1-\delta_{i}\right)^{p}\right)^{\omega_{i}}, \\
& u^{\prime}=\prod_{i=1}^{n}\left(\left(1+(\varepsilon-1) \eta_{i}\right)^{p}-\left(1-\eta_{i}\right)^{p}\right)^{\omega_{i}}, \\
& v^{\prime}=\prod_{i=1}^{n}\left(\left(1+(\varepsilon-1) \eta_{i}\right)^{p}+\left(\varepsilon^{2}-1\right)\left(1-\eta_{i}\right)^{p}\right)^{\omega_{i}} .
\end{aligned}
$$

(2) When $p=1, q=0$, then the operator of MVNLNWBMH defined by (18) will be reduced to the multivalued neutrosophic linguistic Hamacher weighted 
arithmetic average (MVNLHWAA) operator shown as below.

$$
\begin{gathered}
\operatorname{MVNLhWAA}\left(a_{1}, a_{2}, \ldots, a_{n}\right)=\bigoplus_{i=1}^{n} \omega_{i} a_{i}=\left\langle s_{\sum_{i=1}^{n} \omega_{i} \theta\left(a_{i}\right)},\right. \\
\left(\bigcup_{\gamma_{i} \in \widetilde{T}\left(a_{i}\right)}\left\{\frac{\prod_{i=1}^{n}\left(1+(\varepsilon-1) \gamma_{i}\right)^{\omega_{i}}-\prod_{i=1}^{n}\left(1-\gamma_{i}\right)^{\omega_{i}}}{\prod_{i=1}^{n}\left(1+(\varepsilon-1) \gamma_{i}\right)^{\omega_{i}}+(\varepsilon-1) \prod_{i=1}^{n}\left(1-\gamma_{i}\right)^{\omega_{i}}}\right\},\right. \\
\bigcup_{\delta_{i} \in \widetilde{I}\left(a_{i}\right)}\left\{\frac{\varepsilon \prod_{i=1}^{n} \delta_{i}^{\omega_{i}}}{(\varepsilon-1) \prod_{i=1}^{n} \delta_{i}^{\omega_{i}}+\prod_{i=1}^{n}\left(\varepsilon-(\varepsilon-1) \delta_{i}\right)^{\omega_{i}}}\right\}, \\
\left.\left.\bigcup_{\eta_{i} \in \widetilde{F}\left(a_{i}\right)}\left\{\frac{\varepsilon \prod_{i=1}^{n} \eta_{i}^{\omega_{i}}}{(\varepsilon-1) \prod_{i=1}^{n} \eta_{i}{ }_{i}^{\omega_{i}}+\prod_{i=1}^{n}\left(\varepsilon-(\varepsilon-1) \eta_{i}\right)^{\omega_{i}}}\right\}\right)\right\rangle .
\end{gathered}
$$

If $\varepsilon=1$, the MVNLHWAA operator will be reduced to MVNLWAA operator shown as below.

$$
\begin{aligned}
& \operatorname{MVnlwaA}\left(a_{1}, a_{2}, \ldots, a_{n}\right)=\left\langle s_{\sum_{i=1}^{n} \omega_{i} \theta\left(a_{i}\right)},\right. \\
& \left(\bigcup_{\gamma_{i} \in \widetilde{T}\left(a_{i}\right)}\left\{1-\prod_{i=1}^{n}\left(1-\gamma_{i}\right)^{\omega_{i}}\right\}, \bigcup_{\delta_{i} \in \widetilde{I}\left(a_{i}\right)}\left\{\prod_{i=1}^{n} \delta_{i}^{\omega_{i}}\right\},\right. \\
& \left.\left.\bigcup_{\eta_{i} \in \widetilde{F}\left(a_{i}\right)}\left\{\prod_{i=1}^{n} \eta_{i}^{\omega_{i}}\right\}\right)\right\rangle .
\end{aligned}
$$

If $\varepsilon=2$, the MVNLHWAA operator will be reduced to the multivalued neutrosophic linguistic Einstein weighted arithmetic average (MVNLEWAA) operator shown as follows:

$$
\begin{aligned}
& \operatorname{MVNLEWAA}\left(a_{1}, a_{2}, \ldots, a_{n}\right)=\left\langle s_{\sum_{i=1}^{n} \omega_{i} \theta\left(a_{i}\right)},\right. \\
& \left(\bigcup_{\gamma_{i} \in \widetilde{T}\left(a_{i}\right)}\left\{\frac{\prod_{i=1}^{n}\left(1+\gamma_{i}\right)^{\omega_{i}}-\prod_{i=1}^{n}\left(1-\gamma_{i}\right)^{\omega_{i}}}{\prod_{i=1}^{n}\left(1+\gamma_{i}\right)^{\omega_{i}}+\prod_{i=1}^{n}\left(1-\gamma_{i}\right)^{\omega_{i}}}\right\},\right. \\
& \bigcup_{\delta_{i} \in \widetilde{I}\left(a_{i}\right)}\left\{\frac{2 \prod_{i=1}^{n} \delta_{i}^{\omega_{i}}}{\prod_{i=1}^{n}\left(2-\delta_{i}\right)^{\omega_{i}}+\prod_{i=1}^{n} \delta_{i}^{\omega_{i}}}\right\}, \\
& \left.\left.\bigcup_{\eta_{i} \in \widetilde{F}\left(a_{i}\right)}\left\{\frac{2 \prod_{i=1}^{n} \eta_{i}^{\omega_{i}}}{\prod_{i=1}^{n}\left(2-\eta_{i}\right)^{\omega_{i}}+\prod_{i=1}^{n} \eta_{i}^{\omega_{i}}}\right\}\right)\right\rangle .
\end{aligned}
$$

(3) If $p \rightarrow 0, q=0$, then the MVNLNWBMH operator defined by (18) will be reduced to the multivalued neutrosophic linguistic Hamacher weighted geometric average (MVNLHWGA) operator shown as below.

$$
\begin{aligned}
& \operatorname{MVNLhWGA}\left(a_{1}, a_{2}, \ldots, a_{n}\right)=\bigotimes_{i=1}^{n} a_{i}^{\omega_{i}}=\left\langle s_{\prod_{i=1}^{n} \theta^{\omega_{i}}\left(a_{i}\right)},\right. \\
& \left(\bigcup_{\gamma_{i} \in \widetilde{T}\left(a_{i}\right)}\left\{\frac{\varepsilon \prod_{i=1}^{n} \gamma_{i}^{\omega_{i}}}{(\varepsilon-1) \prod_{i=1}^{n} \gamma_{i}^{\omega_{i}}+\prod_{i=1}^{n}\left(\varepsilon-(\varepsilon-1) \gamma_{i}\right)^{\omega_{i}}}\right\},\right. \\
& \bigcup_{\delta_{i} \in \widetilde{I}\left(a_{i}\right)}\left\{\frac{\prod_{i=1}^{n}\left(1+(\varepsilon-1) \delta_{i}\right)^{\omega_{i}}-\prod_{i=1}^{n}\left(1-\delta_{i}\right)^{\omega_{i}}}{\prod_{i=1}^{n}\left(1+(\varepsilon-1) \delta_{i}\right)^{\omega_{i}}+(\varepsilon-1) \prod_{i=1}^{n}\left(1-\delta_{i}\right)^{\omega_{i}}}\right\}, \\
& \left.\left.\bigcup_{\eta_{i} \in \widetilde{E}\left(a_{i}\right)}\left\{\frac{\prod_{i=1}^{n}\left(1+(\varepsilon-1) \eta_{i}\right)^{\omega_{i}}-\prod_{i=1}^{n}\left(1-\eta_{i}\right)^{\omega_{i}}}{\prod_{i=1}^{n}\left(1+(\varepsilon-1) \eta_{i}\right)^{\omega_{i}}+(\varepsilon-1) \prod_{i=1}^{n}\left(1-\eta_{i}\right)^{\omega_{i}}}\right\}\right)\right\rangle .
\end{aligned}
$$

If $\varepsilon=1$, the MVNLHWGA operator will be reduced to MVNLWGA operator shown as below.

$$
\begin{aligned}
& \operatorname{MVNLWGA}\left(a_{1}, a_{2}, \ldots, a_{n}\right)=\left\langle s_{\prod_{i=1}^{n} \theta^{\omega_{i}\left(a_{i}\right)},}\right. \\
& \left(\bigcup_{\gamma_{i} \in \widetilde{T}\left(a_{i}\right)}\left\{\prod_{i=1}^{n} \gamma_{i}^{\omega_{i}}\right\}, \bigcup_{\delta_{i} \in \widetilde{I}\left(a_{i}\right)}\left\{1-\prod_{i=1}^{n}\left(1-\delta_{i}\right)^{\omega_{i}}\right\},\right. \\
& \left.\left.\bigcup_{\eta_{i} \in \widetilde{F}\left(a_{i}\right)}\left\{1-\prod_{i=1}^{n}\left(1-\eta_{i}\right)^{\omega_{i}}\right\}\right)\right\rangle .
\end{aligned}
$$

If $\varepsilon=2$, the MVNLHWGA operator will be reduced to the multivalued neutrosophic linguistic Einstein weighted geometric average (MVNLEWGA) operator shown as below.

$$
\begin{gathered}
\operatorname{MVNLEWGA}\left(a_{1}, a_{2}, \ldots, a_{n}\right)=\left\langle s_{\prod_{i=1}^{n} \theta^{\omega_{i}}\left(a_{i}\right)},\right. \\
\left(\bigcup_{\gamma_{i} \in \widetilde{T}\left(a_{i}\right)}\left\{\frac{2 \prod_{i=1}^{n} \gamma_{i}^{\omega_{i}}}{\prod_{i=1}^{n}\left(2-\gamma_{i}\right)^{\omega_{i}}+\prod_{i=1}^{n} \gamma_{i}^{\omega_{i}}}\right\},\right. \\
\bigcup_{\delta_{i} \in \widetilde{I}\left(a_{i}\right)}\left\{\frac{\prod_{i=1}^{n}\left(1+\delta_{i}\right)^{\omega_{i}}-\prod_{i=1}^{n}\left(1-\delta_{i}\right)^{\omega_{i}}}{\prod_{i=1}^{n}\left(1+\delta_{i}\right)^{\omega_{i}}+\prod_{i=1}^{n}\left(1-\delta_{i}\right)^{\omega_{i}}}\right\}, \\
\left.\left.\bigcup_{\eta_{i} \in \widetilde{F}\left(a_{i}\right)}\left\{\frac{\prod_{i=1}^{n}\left(1+\eta_{i}\right)^{\omega_{i}}-\prod_{i=1}^{n}\left(1-\eta_{i}\right)^{\omega_{i}}}{\prod_{i=1}^{n}\left(1+\eta_{i}\right)^{\omega_{i}}+\prod_{i=1}^{n}\left(1-\eta_{i}\right)^{\omega_{i}}}\right\}\right)\right\rangle .
\end{gathered}
$$

(4) If $\varepsilon=1$, then the MVNLNWBMH operator defined by (18) will be reduced to the MVNLNWBM operator shown as below.

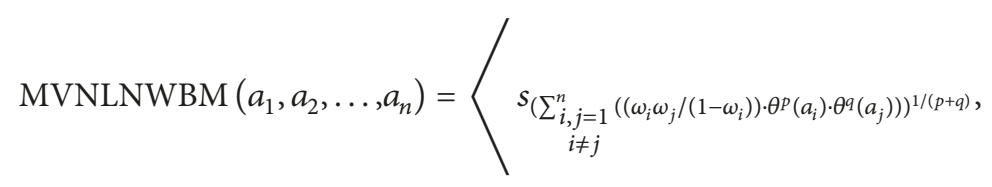




$$
\begin{gathered}
\left(\bigcup_{\gamma_{i} \in \widetilde{T}\left(a_{i}\right), \gamma_{j} \in \widetilde{T}\left(a_{j}\right)}\left\{\left(1-\prod_{\substack{i, j=1 \\
i \neq j}}^{n}\left(1-\gamma_{i}^{p} \gamma_{j}^{q}\right)^{\omega_{i} \omega_{j} /\left(1-\omega_{i}\right)}\right)^{1 /(p+q)}\right\},\right. \\
\bigcup_{\delta_{i} \in \widetilde{I}\left(a_{i}\right), \delta_{j} \in \widetilde{I}\left(a_{j}\right)}\left\{1-\left(1-\prod_{\substack{i, j=1 \\
i \neq j}}^{n}\left(1-\left(1-\delta_{i}\right)^{p}\left(1-\delta_{j}\right)^{q}\right)^{\omega_{i} \omega_{j} /\left(1-\omega_{i}\right)}\right)^{1 /(p+q)}\right\}, \\
\left.\bigcup_{\eta_{i} \in \widetilde{F}\left(a_{i}\right), \eta_{j} \in \tilde{F}\left(a_{j}\right)}\left\{1-\left(\begin{array}{c}
1-\prod_{\substack{i, j=1 \\
i \neq j}}^{n}\left(1-\left(1-\eta_{i}\right)^{p}\left(1-\eta_{j}\right)^{q}\right)^{\omega_{i} \omega_{j} /\left(1-\omega_{i}\right)} \\
\underbrace{1 /(p+q)}
\end{array}\right\}\right)\right\rangle
\end{gathered}
$$

(5) If $\varepsilon=2$, then the MVNLNWBMH operator defined by (18) will be reduced to the MVNLNWBME, that is the simplification of multivalued neutrosophic linguistic NWBM Einstein.

$$
\begin{aligned}
& \operatorname{MVNLNWBME}\left(a_{1}, a_{2}, \ldots, a_{n}\right) \\
& =\left\langle s_{\substack{i, j=1 \\
i \neq j}}^{n}\left(\left(\omega_{i} \omega_{j} /\left(1-\omega_{i}\right)\right) \cdot \theta^{p}\left(a_{i}\right) \cdot \theta^{q}\left(a_{j}\right)\right)\right)^{1 /(p+q)}, \\
& \left(\bigcup_{\gamma_{i} \in \widetilde{T}\left(a_{i}\right), \gamma_{j} \in \widetilde{T}\left(a_{j}\right)}\left\{\frac{2(x-y)^{1 /(p+q)}}{(x+3 y)^{1 /(p+q)}+(x-y)^{1 /(p+q)}}\right\},\right. \\
& \bigcup_{\delta_{i} \in \widetilde{I}\left(a_{i}\right), \delta_{j} \in \widetilde{I}\left(a_{j}\right)}\left\{\frac{(g+3 h)^{1 /(p+q)}-(g-h)^{1 /(p+q)}}{(g+3 h)^{1 /(p+q)}+(g-h)^{1 /(p+q)}}\right\}, \\
& \left.\left.\bigcup_{\eta_{i} \in \widetilde{F}\left(a_{i}\right), \eta_{j} \in \widetilde{F}\left(a_{j}\right)}\left\{\frac{(v+3 u)^{1 /(p+q)}-(v-u)^{1 /(p+q)}}{(v+3 u)^{1 /(p+q)}+(v-u)^{1 /(p+q)}}\right\}\right)\right\rangle .
\end{aligned}
$$

where

$$
\begin{aligned}
x= & \prod_{\substack{i, j=1 \\
i \neq j}}^{n}\left(\left(2-\gamma_{i}\right)^{p}\left(2-\gamma_{j}\right)^{q}+3 \gamma_{i}^{p} \gamma_{j}^{q}\right)^{\omega_{i} \omega_{j} /\left(1-\omega_{i}\right)}, \\
y= & \prod_{\substack{i, j=1 \\
i \neq j}}^{n}\left(\left(2-\gamma_{i}\right)^{p}\left(2-\gamma_{j}\right)^{q}-\gamma_{i}^{p} \gamma_{j}^{q}\right)^{\omega_{i} \omega_{j} /\left(1-\omega_{i}\right)}, \\
h= & \prod_{\substack{i, j=1 \\
i \neq j}}^{n}\left(\left(1+\delta_{i}\right)^{p}\left(1+\delta_{j}\right)^{q}\right. \\
& \left.-\left(1-\delta_{i}\right)^{p}\left(1-\delta_{j}\right)^{q}\right)^{\omega_{i} \omega_{j} /\left(1-\omega_{i}\right)}, \\
g= & \prod_{\substack{i, j=1 \\
i \neq j}}^{n}\left(\left(1+\delta_{i}\right)^{p}\left(1+\delta_{j}\right)^{q}\right. \\
& \left.+3\left(1-\delta_{i}\right)^{p}\left(1-\delta_{j}\right)^{q}\right)^{\omega_{i} \omega_{j} /\left(1-\omega_{i}\right)},
\end{aligned}
$$

$$
\begin{aligned}
u= & \prod_{\substack{i, j=1 \\
i \neq j}}^{n}\left(\left(1+\eta_{i}\right)^{p}\left(1+\eta_{j}\right)^{q}\right. \\
& \left.-\left(1-\eta_{i}\right)^{p}\left(1-\eta_{j}\right)^{q}\right)^{\omega_{i} \omega_{j} /\left(1-\omega_{i}\right)}, \\
v= & \prod_{\substack{i, j=1 \\
i \neq j}}^{n}\left(\left(1+\eta_{i}\right)^{p}\left(1+\eta_{j}\right)^{q}\right. \\
& \left.+3\left(1-\eta_{i}\right)^{p}\left(1-\eta_{j}\right)^{q}\right)^{\omega_{i} \omega_{j} /\left(1-\omega_{i}\right)} .
\end{aligned}
$$

From the above analysis, we can obtain that the MVNLNWBMH operator is more generalized.

\section{The Multiple Criteria Decision Making Approach Based on the MVNLNWBMH Operator}

The proposed MVNLNWBMH operator is presented to cope with MCDM problem under multivalued neutrosophic linguistic environment in this subsection.

Suppose that $A=\left\{A_{1}, A_{2}, \ldots, A_{m}\right\}$ represent $m$ alternatives and $C=\left\{C_{1}, C_{2}, \ldots, C_{n}\right\}$ represent $n$ criteria. Let $\omega=\left\{\omega_{1}, \omega_{2}, \ldots, \omega_{n}\right\}$ be the corresponding weights of criteria, where $w_{j} \geq 0(j=1,2, \ldots, n)$ and $\sum_{j=1}^{n} w_{j}=1$. The evaluation value of the criteria $C_{j}(j=1,2, \ldots, n)$ regarding the alternative $A_{i}(i=1,2, \ldots, m)$ is provided by experts. Each value is represented by MVNLNNs. Suppose that $R=\left[a_{i j}\right]_{m \times n}$ is the multivalued neutrosophic linguistic decision matrix, where $a_{i j}=\left\langle s_{\theta\left(a_{i j}\right)},\left(\widetilde{T}\left(a_{i j}\right), \widetilde{I}\left(a_{i j}\right), \widetilde{F}\left(a_{i j}\right)\right)\right\rangle$ is the evaluation information which represents the assessment value of alternative $A_{i}(i=1,2, \ldots, m)$ on criteria $C_{j}(j=$ $1,2, \ldots, n)$ with respect to the linguistic value $s_{\theta\left(a_{i j}\right)}$, where $\widetilde{T}\left(a_{i j}\right)$ indicates the satisfaction degree, $\widetilde{I}\left(a_{i j}\right)$ indicates the indeterminacy degree, and $\widetilde{F}\left(a_{i j}\right)$ indicates the dissatisfaction degree. 
Then, the main method for ranking and selecting the best alternative is presented below.

Step 1 (the decision matrix is normalized). Generally, criteria in MCDM problems consist of two types: maximum type and minimum type; the minimum type should be transformed into the maximum type for eliminating the influence of distinguished types. Suppose that $R=\left[a_{i j}\right]_{m \times n}$ is the original decision matrix, which can be normalized as follows:

$$
b_{i j}= \begin{cases}a_{i j}, & \text { for maximizing criteria } \\ \left\langle s_{l-\theta\left(a_{i j}\right)},\left(\widetilde{T}\left(a_{i j}\right), \widetilde{I}\left(a_{i j}\right), \widetilde{F}\left(a_{i j}\right)\right)\right\rangle, & \text { for maximizing criteria. }\end{cases}
$$

Thus, the normalized matrix $B=\left[b_{i j}\right]_{m \times n}$ is gained.

Step 2 (the comprehensive value of each alternative is calculated). The comprehensive value represented by $a_{i}(i=$ $1,2, \ldots, m)$ can be obtained by utilizing the MVNLNWBMH operator in Definition 11, which can aggregate the overall value for each alternative with respect to all criteria.

Step 3 (the compared values of three functions are calculated). According to the equations given in Definition 9, the score value denoted by $E\left(a_{i}\right)$, the accuracy value denoted by $H\left(a_{i}\right)$, and the certainty value denoted by $C\left(a_{i}\right)$ can be obtained.

Step 4 (the alternatives are selected). Based on Definition 10, all alternatives $A_{i}(i=1,2, \ldots, m)$ can be ranked on the basis of $E\left(a_{i}\right), H\left(a_{i}\right)$, and $C\left(a_{i}\right)$, and the best alternative(s) can be selected.

\section{A Numerical Example}

In order to validate the effectiveness and practicality of the novel approach, an investment project is adapted from Ye.
An investment company wants to expand its business. Four alternatives will be chosen: $A_{1}$ represents autocorporation, $A_{2}$ represents food corporation, $A_{3}$ represents computer company corporation, $A_{4}$ represents weapon corporation. Each alternative is evaluated under three criteria: $C_{1}$ denotes risk, $C_{2}$ denotes growth, and $C_{3}$ denotes the impact of environment, where $C_{3}$ is the minimizing criteria. The corresponding weighted vector is $\omega=\{0.35,0.25,0.4\}$. In real situation, the decision maker may hesitate and give several possible values for the satisfaction, indeterminacy, and dissatisfaction regarding the alternative $A_{i}$ corresponding to the criteria $C_{j}$ under the linguistic term set $S$. Therefore, the assessment value is given in the form of MVNLNs, and the linguistic term set is employed as $S=\left\{s_{1}, s_{2}, s_{3}, s_{4}, s_{5}, s_{6}, s_{7}\right\}=$ \{extremely poor, very poor, poor, medium, good, very good, extremely good\}.

The multivalued neutrosophic linguistic decision matrix $R=\left[a_{i j}\right]_{4 \times 3}$ is shown as follows:

$$
\begin{aligned}
R & =\left[a_{i j}\right]_{4 \times 3} \\
= & {\left[\begin{array}{ccc}
\left\langle s_{5},(\{0.3,0.4,0.5\},\{0.1\},\{0.3,0.4\})\right\rangle & \left\langle s_{6},(\{0.5,0.6\},\{0.2,0.3\},\{0.3,0.4\})\right\rangle & \left\langle s_{5},(\{0.2,0.3\},\{0.1,0.2\},\{0.5,0.6\})\right\rangle \\
\left\langle s_{6},(\{0.6,0.7\},\{0.1,0.2\},\{0.2,0.3\})\right\rangle & \left\langle s_{5},(\{0.6,0.7\},\{0.1\},\{0.3\})\right\rangle & \left\langle s_{5},(\{0.6,0.7\},\{0.1,0.2\},\{0.1,0.2\})\right\rangle \\
\left\langle s_{6},(\{0.5,0.6\},\{0.4\},\{0.2,0.3\})\right\rangle & \left\langle s_{5},(\{0.6\},\{0.3\},\{0.4\})\right\rangle & \left\langle s_{4},(\{0.5,0.6\},\{0.1\},\{0.3\})\right\rangle \\
\left\langle s_{4},(\{0.7,0.8\},\{0.1\},\{0.1,0.2\})\right\rangle & \left\langle s_{4},(\{0.6,0.7\},\{0.1\},\{0.2\})\right\rangle & \left\langle s_{6},(\{0.3,0.5\},\{0.2\},\{0.1,0.2,0.3\})\right\rangle
\end{array}\right] . }
\end{aligned}
$$

\subsection{The Procedure Using the Proposed Aggregation Operator}

Step 1 (the decision matrix is normalized). Because $C_{3}$ is the minimizing criteria, which should be converted to the maximizing criteria, then the normalized decision matrix $B=$ $\left[b_{i j}\right]_{m \times n}$ can be obtained as follows:

$$
\begin{aligned}
B= & {\left[b_{i j}\right]_{4 \times 3} } \\
= & {\left[\begin{array}{ccc}
\left\langle s_{5},(\{0.3,0.4,0.5\},\{0.1\},\{0.3,0.4\})\right\rangle & \left\langle s_{6},(\{0.5,0.6\},\{0.2,0.3\},\{0.3,0.4\})\right\rangle & \left\langle s_{2},(\{0.2,0.3\},\{0.1,0.2\},\{0.5,0.6\})\right\rangle \\
\left\langle s_{6},(\{0.6,0.7\},\{0.1,0.2\},\{0.2,0.3\})\right\rangle & \left\langle s_{5},(\{0.6,0.7\},\{0.1\},\{0.3\})\right\rangle & \left\langle s_{2},(\{0.6,0.7\},\{0.1,0.2\},\{0.1,0.2\})\right\rangle \\
\left\langle s_{6},(\{0.5,0.6\},\{0.4\},\{0.2,0.3\})\right\rangle & \left\langle s_{5},(\{0.6\},\{0.3\},\{0.4\})\right\rangle & \left\langle s_{3},(\{0.5,0.6\},\{0.1\},\{0.3\})\right\rangle \\
\left\langle s_{4},(\{0.7,0.8\},\{0.1\},\{0.1,0.2\})\right\rangle & \left\langle s_{4},(\{0.6,0.7\},\{0.1\},\{0.2\})\right\rangle & \left\langle s_{1},(\{0.3,0.5\},\{0.2\},\{0.1,0.2,0.3\})\right\rangle
\end{array}\right] . }
\end{aligned}
$$


Step 2 (the comprehensive value of each alternative is calculated). Derive the comprehensive value $a_{i}(i=1,2, \ldots, m)$ of each alternative $A_{i}(i=1,2, \ldots, m)$ by using the
MVNLNWBMH operator presented in Definition 11. Here let $p=q=1, \varepsilon=1$. The MVNLNWBMH operator is shown as below:

$$
\begin{aligned}
& \operatorname{MVNLNWBMH}\left(a_{1}, a_{2}, \ldots, a_{n}\right)=\left\langle s_{\substack{i, j=1 \\
i \neq j}}^{n}\left(\left(\omega_{i} \omega_{j} /\left(1-\omega_{i}\right)\right) \cdot \theta\left(a_{i}\right) \cdot \theta\left(a_{j}\right)\right)\right)^{1 / 2}, \\
& \left(\bigcup_{\gamma_{i} \in \widetilde{T}\left(a_{i}\right), \gamma_{j} \in \widetilde{T}\left(a_{j}\right)}\left\{\left(1-\prod_{\substack{i, j=1 \\
i \neq j}}^{n}\left(1-\gamma_{i} \gamma_{j}\right)^{\omega_{i} \omega_{j} /\left(1-\omega_{i}\right)}\right)^{1 / 2}\right\},\right. \\
& \bigcup_{\delta_{i} \in \widetilde{I}\left(a_{i}\right), \delta_{j} \in \widetilde{I}\left(a_{j}\right)}\left\{1-\left(1-\prod_{\substack{i, j=1 \\
i \neq j}}^{n}\left(1-\left(1-\delta_{i}\right)\left(1-\delta_{j}\right)\right)^{\omega_{i} \omega_{j} /\left(1-\omega_{i}\right)}\right)^{1 / 2}\right\}, \\
& \left.\bigcup_{\eta_{i} \in \widetilde{F}\left(a_{i}\right), \eta_{j} \in \widetilde{F}\left(a_{j}\right)}\left\{1-\left(1-\prod_{\substack{i, j=1 \\
i \neq j}}^{n}\left(1-\left(1-\eta_{i}\right)\left(1-\eta_{j}\right)\right)^{\omega_{i} \omega_{j} /\left(1-\omega_{i}\right)}\right)^{1 / 2}\right\}\right)
\end{aligned}
$$

And we have

$$
\begin{aligned}
a_{1}= & \left\langle s_{3.9529},(\{0.3088,0.3515,0.3309,\right. \\
& 0.3752,0.3429,0.3869,0.3671, \\
& 0.4118,0.3746,0.4199,0.4009,0.4463\}, \\
& \{0.1255,0.1645,0.1482,0.19\}, \\
& \{0.3768,0.4171,0.4049,0.446,0.4109, \\
& 0.4511,0.4382,0.4783\})\rangle ; \\
a_{2}= & \left\langle s_{3.9904},(\{0.6,0.6368,0.6275,0.6648,\right. \\
& 0.6345,0.6727,0.6622,0.7\}, \\
& \{0.1,0.1363,0.1335,0.1714\}, \\
& \{0.1882,0.2268,0.2261,0.2629\})\rangle ; \\
a_{3}= & \left\langle s_{4.4850},(\{0.5272,0.5644,0.5618,0.6\},\right. \\
& \{0.263\},\{0.2918,0.3273\})\rangle ; \\
a_{4}= & \left\langle s_{2.6487},(\{0.5107,0.5951,0.5406,\right. \\
& 0.6232,0.5415,0.6275,0.5743,0.6569\}, \\
& \{0.1363\},\{0.1255,0.1645, \\
& 0.2021,0.1623,0.2,0.2373\})\rangle .
\end{aligned}
$$

Step 3 (the compared values of three functions are calculated). By using the equations in Definition 9, we can obtain $E\left(a_{i}\right)$, $H\left(a_{i}\right)$, and $C\left(a_{i}\right)(i=1,2,3,4)$ as follows:

$$
\begin{aligned}
& E\left(a_{1}\right)=s_{2.3605}, \\
& H\left(a_{1}\right)=s_{-0.2036} \\
& C\left(a_{1}\right)=s_{1.4879} \\
& E\left(a_{2}\right)=s_{3.0440} \\
& H\left(a_{2}\right)=s_{1.6912} \\
& C\left(a_{2}\right)=s_{2.5930} \\
& E\left(a_{3}\right)=s_{2.9763} \\
& H\left(a_{3}\right)=s_{1.1382} \\
& C\left(a_{3}\right)=s_{2.5267} \\
& E\left(a_{4}\right)=s_{2.0002} \\
& H\left(a_{4}\right)=s_{1.0641}, \\
& C\left(a_{4}\right)=s_{1.5461}
\end{aligned}
$$

Step 4 (the alternatives are selected). By using the compared approach in Definition 10, $E\left(a_{2}\right)>E\left(a_{3}\right)>E\left(a_{1}\right)>E\left(a_{4}\right)$ can be obtained, so the final raking of alternatives is $A_{2}>A_{3}>$ $A_{1}>A_{4}$. Apparently, $A_{2}$ is the best one, and $A_{4}$ is the worst one. We do not need to compare the other functions because the values of score function differ.

6.2. Comparison Analysis. We take different value into consideration in Step 2 to select the alternative for discussing the 
TABLE 1: Ranking of alternatives utilizing different $p, q$, and $\varepsilon=1$.

\begin{tabular}{|c|c|c|}
\hline$\varepsilon=1, p, q$ & Score function $E\left(a_{i}\right),(i=1,2,3,4)$ & Ranking \\
\hline$p \rightarrow 0, q=0$ & $E\left(a_{1}\right)=s_{2.1214}, E\left(a_{2}\right)=s_{2.8131}, E\left(a_{3}\right)=s_{2.8732}, E\left(a_{4}\right)=s_{1.7094}$ & $A_{3}>A_{2}>A_{1}>A_{4}$ \\
\hline$p=0.01, q=0$ & $E\left(a_{1}\right)=s_{2.1907}, E\left(a_{2}\right)=s_{2.8460}, E\left(a_{3}\right)=s_{2.9575}, E\left(a_{4}\right)=s_{1.7629}$ & $A_{3}>A_{2}>A_{1}>A_{4}$ \\
\hline$p=0.1, q=0$ & $E\left(a_{1}\right)=s_{2.2165}, E\left(a_{2}\right)=s_{2.8790}, E\left(a_{3}\right)=s_{2.9713}, E\left(a_{4}\right)=s_{1.8005}$ & $A_{3}>A_{2}>A_{1}>A_{4}$ \\
\hline$p=1, q=0$ & $E\left(a_{1}\right)=s_{2.4697}, E\left(a_{2}\right)=s_{3.1970}, E\left(a_{3}\right)=s_{3.1084}, E\left(a_{4}\right)=s_{2.1573}$ & $A_{2}>A_{3}>A_{1}>A_{4}$ \\
\hline$p=2, q=0$ & $E\left(a_{1}\right)=s_{2.7154}, E\left(a_{2}\right)=s_{3.4882}, E\left(a_{3}\right)=s_{3.2507}, E\left(a_{4}\right)=s_{2.4519}$ & $A_{2}>A_{3}>A_{1}>A_{4}$ \\
\hline$p=5, q=0$ & $E\left(a_{1}\right)=s_{3.1768}, E\left(a_{2}\right)=s_{3.9655}, E\left(a_{3}\right)=s_{3.5749}, E\left(a_{4}\right)=s_{2.8445}$ & $A_{2}>A_{3}>A_{1}>A_{4}$ \\
\hline$p=10, q=0$ & $E\left(a_{1}\right)=s_{3.5203}, E\left(a_{2}\right)=s_{4.2619}, E\left(a_{3}\right)=s_{3.8673}, E\left(a_{4}\right)=s_{3.0415}$ & $A_{2}>A_{3}>A_{1}>A_{4}$ \\
\hline$p=0, q=1$ & $E\left(a_{1}\right)=s_{2.6253}, E\left(a_{2}\right)=s_{3.3082}, E\left(a_{3}\right)=s_{3.1563}, E\left(a_{4}\right)=s_{2.2904}$ & $A_{3}>A_{2}>A_{1}>A_{4}$ \\
\hline$p=0.01, q=1$ & $E\left(a_{1}\right)=s_{2.6151}, E\left(a_{2}\right)=s_{3.2980}, E\left(a_{3}\right)=s_{3.1492}, E\left(a_{4}\right)=s_{2.2778}$ & $A_{2}>A_{3}>A_{1}>A_{4}$ \\
\hline$p=0.1, q=1$ & $E\left(a_{1}\right)=s_{2.5377}, E\left(a_{2}\right)=s_{3.2202}, E\left(a_{3}\right)=s_{3.0964}, E\left(a_{4}\right)=s_{2.1839}$ & $A_{2}>A_{3}>A_{1}>A_{4}$ \\
\hline$p=1, q=1$ & $E\left(a_{1}\right)=s_{2.3605}, E\left(a_{2}\right)=s_{3.0440}, E\left(a_{3}\right)=s_{2.9763}, E\left(a_{4}\right)=s_{2.0002}$ & $A_{2}>A_{3}>A_{1}>A_{4}$ \\
\hline$p=2, q=1$ & $E\left(a_{1}\right)=s_{2.4824}, E\left(a_{2}\right)=s_{3.1918}, E\left(a_{3}\right)=s_{3.0464}, E\left(a_{4}\right)=s_{2.1664}$ & $A_{2}>A_{3}>A_{1}>A_{4}$ \\
\hline$p=5, q=1$ & $E\left(a_{1}\right)=s_{2.9208}, E\left(a_{2}\right)=s_{3.6644}, E\left(a_{3}\right)=s_{3.3532}, E\left(a_{4}\right)=s_{2.5870}$ & $A_{2}>A_{3}>A_{1}>A_{4}$ \\
\hline$p=10, q=1$ & $E\left(a_{1}\right)=s_{3.3239}, E\left(a_{2}\right)=s_{4.0427}, E\left(a_{3}\right)=s_{3.6946}, E\left(a_{4}\right)=s_{2.8680}$ & $A_{2}>A_{3}>A_{1}>A_{4}$ \\
\hline$p=0, q=2$ & $E\left(a_{1}\right)=s_{2.8597}, E\left(a_{2}\right)=s_{3.5712}, E\left(a_{3}\right)=s_{3.2871}, E\left(a_{4}\right)=s_{2.5589}$ & $A_{2}>A_{3}>A_{1}>A_{4}$ \\
\hline$p=0.01, q=2$ & $E\left(a_{1}\right)=s_{2.8519}, E\left(a_{2}\right)=s_{3.5630}, E\left(a_{3}\right)=s_{3.2820}, E\left(a_{4}\right)=s_{2.5494}$ & $A_{2}>A_{3}>A_{1}>A_{4}$ \\
\hline$p=0.1, q=2$ & $E\left(a_{1}\right)=s_{2.7889}, E\left(a_{2}\right)=s_{3.4959}, E\left(a_{3}\right)=s_{3.2400}, E\left(a_{4}\right)=s_{2.4731}$ & $A_{2}>A_{3}>A_{1}>A_{4}$ \\
\hline$p=1, q=2$ & $E\left(a_{1}\right)=s_{2.5228}, E\left(a_{2}\right)=s_{3.2087}, E\left(a_{3}\right)=s_{3.0542}, E\left(a_{4}\right)=s_{2.1890}$ & $A_{2}>A_{3}>A_{1}>A_{4}$ \\
\hline$p=2, q=2$ & $E\left(a_{1}\right)=s_{2.5363}, E\left(a_{2}\right)=s_{3.2305}, E\left(a_{3}\right)=s_{3.0440}, E\left(a_{4}\right)=s_{2.2430}$ & $A_{2}>A_{3}>A_{1}>A_{4}$ \\
\hline$p=5, q=2$ & $E\left(a_{1}\right)=s_{2.8535}, E\left(a_{2}\right)=s_{3.5768}, E\left(a_{3}\right)=s_{3.2558}, E\left(a_{4}\right)=s_{2.5581}$ & $A_{2}>A_{3}>A_{1}>A_{4}$ \\
\hline$p=10, q=2$ & $E\left(a_{1}\right)=s_{3.2306}, E\left(a_{2}\right)=s_{3.9375}, E\left(a_{3}\right)=s_{3.5836}, E\left(a_{4}\right)=s_{2.8212}$ & $A_{2}>A_{3}>A_{1}>A_{4}$ \\
\hline
\end{tabular}

impact of different values $p, q$, and $\varepsilon$. The comparisons are presented in Tables 1 and 2 .

In Table 1 , we take the parameter value $\varepsilon=1$, which is based on algebraic operation, and the MVNLNWBM operator is applied. In Table 2, we take the parameter value $\varepsilon=2$, which is based on Einstein operation, and the MVNLNWBME operator is applied. As we can see from Tables 1 and 2 , the ordering of alternatives taking different parameters $p$, $q$, and $\varepsilon$ may be different, because the different parameters will cause different score function value. However, $A_{2}$ or $A_{3}$ is always the best selection, and $A_{4}$ is always the worst selection. Whether $\varepsilon=1$ or $\varepsilon=2$, the same ranking result is obtained with regard to the same parameter value $p$ and the same parameter value $q$ except for one situation in which $p=0, q=1$, and $\varepsilon=1$. Specially, if $p \rightarrow 0, q=0$, MVNLNWBMH will reduce to MVNLHWGA operator. If $p \rightarrow 0, q=0$, and $\varepsilon=1$, MVNLHWGA will reduce to MVNLWGA operator. When $p=1, q=0$, MVNLNWBMH will reduce to MVNLHWAA operator. If $p=1, q=0$, and $\varepsilon=1$, MVNLHWAA will reduce to MVNLWAA operator. If $\varepsilon=1$ in Table 1 , the ranking results on the basis of the MVNLWGA and MVNLWAA operators differ, which is due to the two operators emphasis on different major points, and the same situation happens in Table 2 . When the parameters $q=0$ and $p \rightarrow 0, p=0.01, p=0.1$, respectively, the rankings are identical in two Tables, the ranking order is always $A_{3}>A_{2}>A_{1}>A_{4}$. When $p$ and $q$ are assigned the other values in two Tables, the ranking order is changed, and the result is $A_{2}>A_{3}>A_{1}>A_{4}$. That is, the best selection is from $A_{3}$ to $A_{2}$ except for one situation where $p=0, q=1$ in Table 1.
For illustrating the effectiveness and flexibility of the novel approach, the method in literature is adopted in multivalued neutrosophic linguistic environment in this paper, and the same ranking orders are obtained in [9] where the SVNHFWA and SVNHFWG operators are adopted to fuse single-valued neutrosophic hesitant fuzzy information. When $\varepsilon=1, p \rightarrow 0, q=0$ and $\varepsilon=1, p=0, q=$ 0 , the two operators are special cases of MVNLNWBMH operator. Therefore, novel operator in this paper has better flexibility and generalization. In actual cases, the decision makers can assign different parameter values $\varepsilon$, $p$, and $q$. Generally, for convenience, we can set $\varepsilon=p=1$, which can not only simplify the calculation, but also consider the interrelationship of multiple values.

\section{Conclusions}

In this paper, the MVNLSs are proposed by combining the MVNS and LS, which not only describe linguistic terms, but also give the quantitative value of three membership degrees concerning the linguistic variables, which has better flexibility in expressing the decision information. Moreover, NWBM is a useful operator which has the trait of taking into account the interrelationship of different arguments and overcomes drawbacks of nonreducibility and nonidempotency. Hamacher operations are the extension of algebraic and Einstein operations, which is more general. Considering these advantages, we have developed Hamacher operational laws for MVNLNs and extended the NWBM to fuse MVNL information. Thus, the MVNLNWBMH operator is proposed, which is appropriate to deal with MVNL information. Some 
TABLE 2: Ranking of alternatives utilizing different $p, q$, and $\varepsilon=2$.

\begin{tabular}{|c|c|c|}
\hline$\varepsilon=2, p, q$ & Score function $E\left(a_{i}\right),(i=1,2,3,4)$ & Ranking \\
\hline$p \rightarrow 0, q=0$ & $E\left(a_{1}\right)=s_{2.1344}, E\left(a_{2}\right)=s_{2.8137}, E\left(a_{3}\right)=s_{2.8863}, E\left(a_{4}\right)=s_{1.7187}$ & $A_{3}>A_{2}>A_{1}>A_{4}$ \\
\hline$p=0.01, q=0$ & $E\left(a_{1}\right)=s_{2.1944}, E\left(a_{2}\right)=s_{2.8478}, E\left(a_{3}\right)=s_{2.9631}, E\left(a_{4}\right)=s_{1.7661}$ & $A_{3}>A_{2}>A_{1}>A_{4}$ \\
\hline$p=0.1, q=0$ & $E\left(a_{1}\right)=s_{2.2171}, E\left(a_{2}\right)=s_{2.8803}, E\left(a_{3}\right)=s_{2.9752}, E\left(a_{4}\right)=s_{1.8021}$ & $A_{3}>A_{2}>A_{1}>A_{4}$ \\
\hline$p=1, q=0$ & $E\left(a_{1}\right)=s_{2.4553}, E\left(a_{2}\right)=s_{3.1938}, E\left(a_{3}\right)=s_{3.0982}, E\left(a_{4}\right)=s_{2.1483}$ & $A_{2}>A_{3}>A_{1}>A_{4}$ \\
\hline$p=2, q=0$ & $E\left(a_{1}\right)=s_{2.7142}, E\left(a_{2}\right)=s_{3.4825}, E\left(a_{3}\right)=s_{3.2361}, E\left(a_{4}\right)=s_{2.4453}$ & $A_{2}>A_{3}>A_{1}>A_{4}$ \\
\hline$p=5, q=0$ & $E\left(a_{1}\right)=s_{3.2212}, E\left(a_{2}\right)=s_{3.9697}, E\left(a_{3}\right)=s_{3.5908}, E\left(a_{4}\right)=s_{2.8608}$ & $A_{2}>A_{3}>A_{1}>A_{4}$ \\
\hline$p=10, q=0$ & $E\left(a_{1}\right)=s_{3.5770}, E\left(a_{2}\right)=s_{4.2923}, E\left(a_{3}\right)=s_{3.9217}, E\left(a_{4}\right)=s_{3.0737}$ & $A_{2}>A_{3}>A_{1}>A_{4}$ \\
\hline$p=0, q=1$ & $E\left(a_{1}\right)=s_{2.6103}, E\left(a_{2}\right)=s_{3.3048}, E\left(a_{3}\right)=s_{3.1457}, E\left(a_{4}\right)=s_{2.2818}$ & $A_{2}>A_{3}>A_{1}>A_{4}$ \\
\hline$p=0.01, q=1$ & $E\left(a_{1}\right)=s_{2.6011}, E\left(a_{2}\right)=s_{3.2948}, E\left(a_{3}\right)=s_{3.1394}, E\left(a_{4}\right)=s_{2.2700}$ & $A_{2}>A_{3}>A_{1}>A_{4}$ \\
\hline$p=0.1, q=1$ & $E\left(a_{1}\right)=s_{2.5312}, E\left(a_{2}\right)=s_{3.2188}, E\left(a_{3}\right)=s_{3.0925}, E\left(a_{4}\right)=s_{2.1809}$ & $A_{2}>A_{3}>A_{1}>A_{4}$ \\
\hline$p=1, q=1$ & $E\left(a_{1}\right)=s_{2.3702}, E\left(a_{2}\right)=s_{3.0464}, E\left(a_{3}\right)=s_{2.9849}, E\left(a_{4}\right)=s_{2.0055}$ & $A_{2}>A_{3}>A_{1}>A_{4}$ \\
\hline$p=2, q=1$ & $E\left(a_{1}\right)=s_{2.4994}, E\left(a_{2}\right)=s_{3.1940}, E\left(a_{3}\right)=s_{3.0556}, E\left(a_{4}\right)=s_{2.1751}$ & $A_{2}>A_{3}>A_{1}>A_{4}$ \\
\hline$p=5, q=1$ & $E\left(a_{1}\right)=s_{2.9637}, E\left(a_{2}\right)=s_{3.6734}, E\left(a_{3}\right)=s_{3.3833}, E\left(a_{4}\right)=s_{2.6091}$ & $A_{2}>A_{3}>A_{1}>A_{4}$ \\
\hline$p=10, q=1$ & $E\left(a_{1}\right)=s_{3.3787}, E\left(a_{2}\right)=s_{4.0718}, E\left(a_{3}\right)=s_{3.7516}, E\left(a_{4}\right)=s_{2.9010}$ & $A_{2}>A_{3}>A_{1}>A_{4}$ \\
\hline$p=0, q=2$ & $E\left(a_{1}\right)=s_{2.8577}, E\left(a_{2}\right)=s_{3.5652}, E\left(a_{3}\right)=s_{3.2720}, E\left(a_{4}\right)=s_{2.5525}$ & $A_{2}>A_{3}>A_{1}>A_{4}$ \\
\hline$p=0.01, q=2$ & $E\left(a_{1}\right)=s_{2.8506}, E\left(a_{2}\right)=s_{3.5572}, E\left(a_{3}\right)=s_{3.2675}, E\left(a_{4}\right)=s_{2.5435}$ & $A_{2}>A_{3}>A_{1}>A_{4}$ \\
\hline$p=0.1, q=2$ & $E\left(a_{1}\right)=s_{2.7919}, E\left(a_{2}\right)=s_{3.4917}, E\left(a_{3}\right)=s_{3.2304}, E\left(a_{4}\right)=s_{2.4707}$ & $A_{2}>A_{3}>A_{1}>A_{4}$ \\
\hline$p=1, q=2$ & $E\left(a_{1}\right)=s_{2.5398}, E\left(a_{2}\right)=s_{3.2108}, E\left(a_{3}\right)=s_{3.0635}, E\left(a_{4}\right)=s_{2.1976}$ & $A_{2}>A_{3}>A_{1}>A_{4}$ \\
\hline$p=2, q=2$ & $E\left(a_{1}\right)=s_{2.5586}, E\left(a_{2}\right)=s_{3.2343}, E\left(a_{3}\right)=s_{3.0581}, E\left(a_{4}\right)=s_{2.2559}$ & $A_{2}>A_{3}>A_{1}>A_{4}$ \\
\hline$p=5, q=2$ & $E\left(a_{1}\right)=s_{2.8950}, E\left(a_{2}\right)=s_{3.5871}, E\left(a_{3}\right)=s_{3.2878}, E\left(a_{4}\right)=s_{2.5824}$ & $A_{2}>A_{3}>A_{1}>A_{4}$ \\
\hline$p=10, q=2$ & $E\left(a_{1}\right)=s_{3.2843}, E\left(a_{2}\right)=s_{3.9650}, E\left(a_{3}\right)=s_{3.6392}, E\left(a_{4}\right)=s_{2.8549}$ & $A_{2}>A_{3}>A_{1}>A_{4}$ \\
\hline
\end{tabular}

desirable properties of the novel operator are discussed in detail, and some special cases are analyzed. Furthermore, the comparison method for MVNLNs is also studied, and the rankings of alternatives affected by different parameters $p, q$, and $\varepsilon$ are also compared. For verifying the novel approach, we successfully applied the approach to an example. The results show the novel approach has the following advantages: the MVNLNWBMH operator is more flexible and more general and can capture the interrelationship among arguments and express decision information more practically; the decision makers can assign appropriate values according to the real situation. In future, we will explore applying the operator to the different domains, for instance, fault diagnosis, machine learning, and medical diagnosis.

As a future possible research, we will extend our research by using the refined neutrosophic set [54]; that is, the truth value $T$ is refined into types of subtruths such as $T 1, T 2$; similarly indeterminacy $I$ is refined into types of subindeterminacies $I 1, I 2$, and so forth; and the subfalsehood $F$ is split into $F 1, F 2$, and so forth.

\section{Conflicts of Interest}

The authors declare that they have no conflicts of interest regarding the publication of this paper.

\section{Acknowledgments}

This paper is supported by the National Natural Science Foundation of China (no. 71371154), the Humanities and Social Sciences Research Project of Ministry of Education of
China (no. 16YJCZH049), Thinking Bank Project of Hubei (no. HBSXK2017055), and the Humanities and Social Sciences Foundation of the Department of Education of Hubei (no. 17Q121).

\section{References}

[1] L. A. Zadeh, "Fuzzy sets," Information and Control, vol. 8, no. 3, pp. 338-353, 1965.

[2] K. T. Atanassov, "Intuitionistic fuzzy sets," Fuzzy Sets and Systems, vol. 20, no. 1, pp. 87-96, 1986.

[3] V. Torra, "Hesitant fuzzy sets," International Journal of Intelligent Systems, vol. 25, no. 6, pp. 529-539, 2010.

[4] F. Smarandache, Neutrosophy, Neutrosophic Set, Logic, Probability and Statistics, University of New Mexico, 1995, http://fs .gallup.unm.edu/neutrosophy.htm.

[5] F. Smarandache, A Unifying Field in Logics. Neutrosophy: Neutrosophic Probability, Set and Logic. Rehoboth, American Research Press, 1998.

[6] H. Wang, F. Smarandache, YQ. Zhang, and R. Sunderraman, Single Valued Neutrosophic Sets. Multisp Multistruct, vol. 4, 2010.

[7] J. Ye, "A multicriteria decision-making method using aggregation operators for simplified neutrosophic sets," Journal of Intelligent \& Fuzzy Systems. Applications in Engineering and Technology, vol. 26, no. 5, pp. 2459-2466, 2014.

[8] H. Wang, F. Smarandache, Y.-Q. Zhang, and R. Sunderraman, Interval Neutrosophic Sets and Logic: Theory and Applications in Computing, Hexis, Phoenix, Ariz, USA, 2005.

[9] J. Ye, "Multiple-attribute decision-making method under a single-valued neutrosophic hesitant fuzzy environment," Journal of Intelligent Systems, vol. 24, no. 1, pp. 23-36, 2014. 
[10] J.-Q. Wang and X.-E. Li, “TODIM method with multi-valued neutrosophic sets," Kongzhi yu Juece/Control and Decision, vol. 30, no. 6, pp. 1139-1142, 2015.

[11] P.-D. Liu and Y.-M. Wang, "Multiple attribute decisionmaking method based on single-valued neutrosophic normalized weighted Bonferroni mean," Neural Computing and Applications, vol. 25, no. 7-8, pp. 2001-2010, 2014.

[12] J. Ye, "Single valued neutrosophic cross-entropy for multicriteria decision making problems," Applied Mathematical Modelling, vol. 38, no. 3, pp. 1170-1175, 2014.

[13] H.-Y. Zhang, J.-Q. Wang, and X.-H. Chen, "Interval neutrosophic sets and their application in multicriteria decision making problems," The Scientific World Journal, vol. 2014, Article ID 645953, 15 pages, 2014.

[14] P. Liu and L. Shi, "The generalized hybrid weighted average operator based on interval neutrosophic hesitant set and its application to multiple attribute decision making," Neural Computing \& Applications, vol. 26, no. 2, pp. 457-471, 2014.

[15] S. Broumi and F. Smarandache, "Correlation coefficient of interval neutrosophic set," Applied Mechanics and Materials, vol. 436, pp. 511-517, 2013.

[16] S. Broumi and F. Smarandache, "Cosine similarity measure of interval valued neutrosophic sets," in Neutrosophic Sets Syst, vol. 5, pp. 15-20, 2014.

[17] S. Broumi and F. Smarandache, "Operations on Interval Neutrosophic Sets," Journal of New Theory, vol. 1, pp. 24-37, 2015.

[18] J. Ye, "Similarity measures between interval neutrosophic sets and their applications in multicriteria decision-making," Journal of Intelligent \& Fuzzy Systems, vol. 26, Article ID 165172, 2014.

[19] J.-J. Peng, J.-Q. Wang, X.-H. Wu, J. Wang, and X.-H. Chen, "Multi-valued Neutrosophic Sets and Power Aggregation Operators with Their Applications in Multi-criteria Group Decisionmaking Problems," International Journal of Computational Intelligence Systems, vol. 8, no. 2, pp. 345-363, 2015.

[20] J. Peng, J. Wang, and X. Wu, "An extension of the ELECTRE approach with multi-valued neutrosophic information," Neural Computing and Applications, vol. 28, no. S1, pp. 1011-1022, 2017.

[21] P. Ji, H.-Y. Zhang, and J.-Q. Wang, "A projection-based TODIM method under multi-valued neutrosophic environments and its application in personnel selection," Neural Computing and Applications, pp. 1-14, 2016.

[22] L. A. Zadeh, "The concept of a linguistic variable and its application to approximate reasoning-I," Information Sciences, vol. 8, no. 3, pp. 199-249, 1975.

[23] J.-q. Wang, J. Wang, Q.-h. Chen, H.-y. Zhang, and X.-h. Chen, "An outranking approach for multi-criteria decision-making with hesitant fuzzy linguistic term sets," Information Sciences, vol. 280, pp. 338-351, 2014.

[24] J. Wang, J. Wang, and H. Zhang, "A likelihood-based TODIM approach based on multi-hesitant fuzzy linguistic information for evaluation in logistics outsourcing," Computers \& Industrial Engineering, vol. 99, pp. 287-299, 2016.

[25] J. Wang, J.-q. Wang, H.-y. Zhang, and X.-h. Chen, "Multicriteria group decision-making approach based on 2-tuple linguistic aggregation operators with multi-hesitant fuzzy linguistic information," International Journal of Fuzzy Systems, vol. 18, no. 1, pp. 81-97, 2016

[26] F. Meng, X. Chen, and Q. Zhang, "Multi-attribute decision analysis under a linguistic hesitant fuzzy environment," Information Sciences, vol. 267, pp. 287-305, 2014.
[27] Z. Tian, J. Wang, J. Wang, and X. Chen, "Multicriteria decisionmaking approach based on gray linguistic weighted Bonferroni mean operator," International Transactions in Operational Research, pp. n/a-n/a, 2015.

[28] J. Ye, "An extended TOPSIS method for multiple attribute group decision making based on single valued neutrosophic linguistic numbers," Journal of Intelligent \& Fuzzy Systems. Applications in Engineering and Technology, vol. 28, no. 1, pp. 247-255, 2015.

[29] S. Broumi and F. Smarandache, "Single valued neutrosophic trapezoid linguistic aggregation operators based multi-attribute decision making," Bulletin of Pure \& Applied Sciences- Mathematics and Statistics, vol. 33, no. 2, pp. 135-155, 2014.

[30] Z.-P. Tian, J. Wang, H.-Y. Zhang, X.-H. Chen, and J.-Q. Wang, "Simplified neutrosophic linguistic normalizedweighted bonferroni mean operator and its application to multi-criteria decision-making problems," Filomat, vol. 30, no. 12, pp. 33393360, 2016.

[31] Z. Tian, J. Wang, H. Zhang, and J. Wang, "Multi-criteria decision-making based on generalized prioritized aggregation operators under simplified neutrosophic uncertain linguistic environment," International Journal of Machine Learning and Cybernetics.

[32] J. Ye, "Some aggregation operators of interval neutrosophic linguistic numbers for multiple attribute decision making," Journal of Intelligent \& Fuzzy Systems. Applications in Engineering and Technology, vol. 27, no. 5, pp. 2231-2241, 2014.

[33] Y.-X. Ma, J.-Q. Wang, J. Wang, and X.-H. Wu, "An interval neutrosophic linguistic multi-criteria group decision-making method and its application in selecting medical treatment options," Neural Computing and Applications, vol. 28, no. 9, pp. 2745-2765, 2017.

[34] S. Broumi, J. Ye, and F. Smarandache, "An extended TOPSIS method for multiple attribute decision making based on interval neutrosophic uncertain linguistic variables," in Neutrosophic Sets Syst, vol. 8, pp. 23-32, An extended TOPSIS method for multiple attribute decision making based on interval neutrosophic uncertain linguistic variables. Neutrosophic Sets Syst, 2015.

[35] R. R. Yager, “Prioritized aggregation operators," International Journal of Approximate Reasoning, vol. 48, no. 1, pp. 263-274, 2008.

[36] Z. S. Xu, "Fuzzy harmonic mean operators," International Journal of Intelligent Systems, vol. 24, no. 2, pp. 152-172, 2009.

[37] J. Ye, "Multiple attribute decision-making method based on the possibility degree ranking method and ordered weighted aggregation operators of interval neutrosophic numbers," Journal of Intelligent \& Fuzzy Systems. Applications in Engineering and Technology, vol. 28, no. 3, pp. 1307-1317, 2015.

[38] H.-X. Sun, H.-X. Yang, J.-Z. Wu, and Y. Ouyang, "Interval neutrosophic numbers Choquet integral operator for multicriteria decision making," Journal of Intelligent \& Fuzzy Systems. Applications in Engineering and Technology, vol. 28, no. 6, pp. 2443-2455, 2015.

[39] P. Liu and Y. Wang, "Interval neutrosophic prioritized OWA operator and its application to multiple attribute decision making," Journal of Systems Science and Complexity, pp. 1-17, 2015.

[40] P. Liu and F. Teng, "Multiple attribute decision making method based on normal neutrosophic generalized weighted power averaging operator," International Journal of Machine Learning and Cybernetics. 
[41] D.-F. Li, "The GOWA operator based approach to multiattribute decision making using intuitionistic fuzzy sets," Mathematical and Computer Modelling, vol. 53, no. 5-6, pp. 1182-1196, 2011.

[42] G. Wei, "Some induced geometric aggregation operators with intuitionistic fuzzy information and their application to group decision making," Applied Soft Computing, vol. 10, no. 2, pp. 423-431, 2010.

[43] C. Bonferroni, "Sulle medie multiple di potenze," Bollettino della Unione Matematica Italiana, vol. 5, pp. 267-270, 1950.

[44] G. Beliakov and S. James, "On extending generalized Bonferroni means to Atanassov orthopairs in decision making contexts," Fuzzy Sets and Systems, vol. 211, pp. 84-98, 2013.

[45] Z. S. Xu and R. R. Yager, "Intuitionistic fuzzy bonferroni means," IEEE Transactions on Systems, Man, and Cybernetics, Part B: Cybernetics, vol. 41, no. 2, pp. 568-578, 2011.

[46] W. Zhou and J.-M. He, "Intuitionistic fuzzy normalized weighted bonferroni mean and its application in multicriteria decision making," Journal of Applied Mathematics, vol. 2012, Article ID 136254, 2012.

[47] P. D. Liu and F. Jin, “The trapezoid fuzzy linguistic Bonferroni mean operators and their application to multiple attribute decision making," Scientia Iranica, vol. 19, no. 6, pp. 1947-1959, 2012.

[48] P. Liu, Y. Chen, and Y. Chu, "Intuitionistic uncertain linguistic weighted bonferroni OWA operator and its application to multiple attribute decision making," Cybernetics and Systems, vol. 45, no. 5, pp. 418-438, 2014.

[49] P. D. Liu, L. L. Rong, Y. C. Chu, and Y. W. Li, "Intuitionistic linguistic weighted bonferroni mean operator and its application to multiple attribute decision making," The Scientific World Journal, vol. 2014, Article ID 545049, 13 pages, 2014.

[50] P. Liu and L. Shi, "Some neutrosophic uncertain linguistic number Heronian mean operators and their application to multi-attribute group decision making," Neural Computing and Applications, vol. 28, no. 5, pp. 1079-1093, 2017.

[51] P. D. Liu, "Some Hamacher aggregation operators based on the interval-valued intuitionistic fuzzy numbers and their application to group decision making," IEEE Transactions on Fuzzy Systems, vol. 22, no. 1, pp. 83-97, 2014.

[52] P. D. Liu, Y. C. Chu, Y. W. Li, and Y. B. Chen, "Some generalized neutrosophic number hamacher aggregation operators and their application to group decision making," International Journal of Fuzzy Systems, vol. 16, no. 2, pp. 242-255, 2014.

[53] Z. S. Xu, "A note on linguistic hybrid arithmetic averaging operator in multiple attribute group decision making with linguistic information," Group Decision and Negotiation, vol. 15, no. 6, pp. 593-604, 2006.

[54] F. Smarandache, n-Valued Refined Neutrosophic Logic and Its Applications in Physics. Progress in Physics, vol. 4, American Research Press, Rehoboth, DE, USA, 1999. 


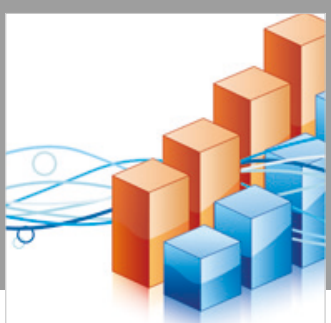

Advances in

Operations Research

\section{-n-m}
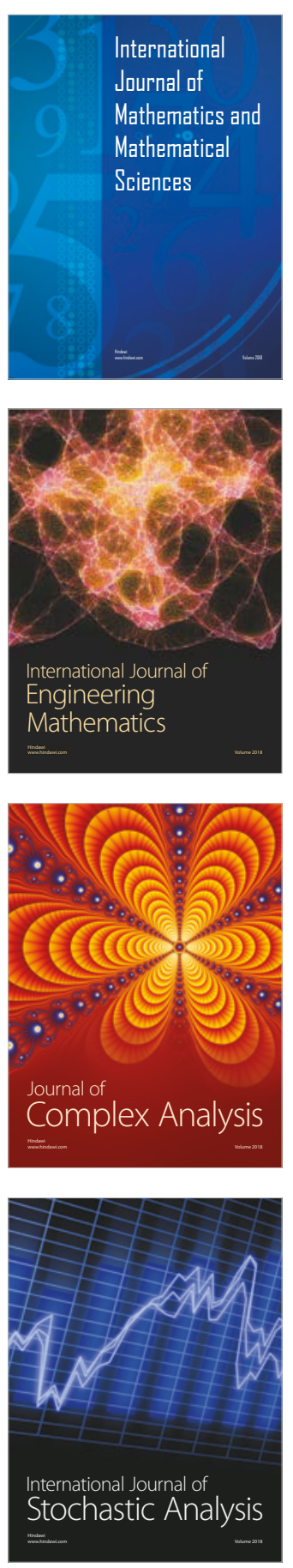
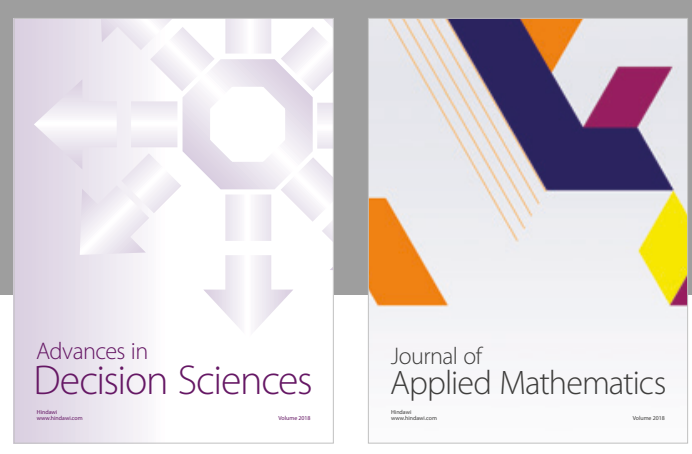

Journal of

Applied Mathematics
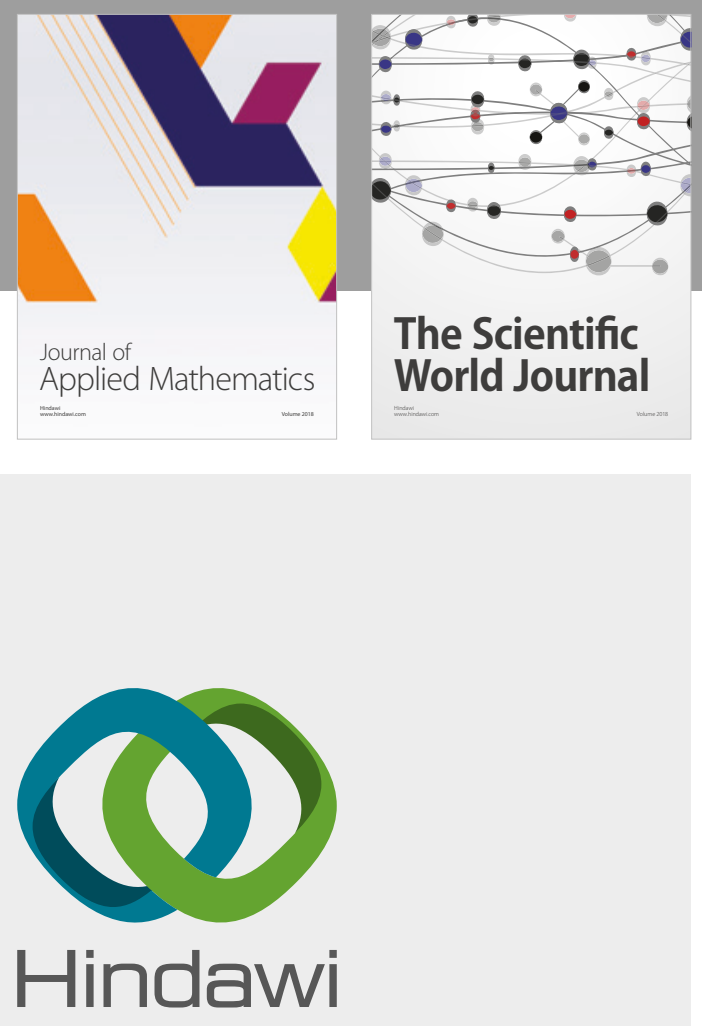

Submit your manuscripts at

www.hindawi.com

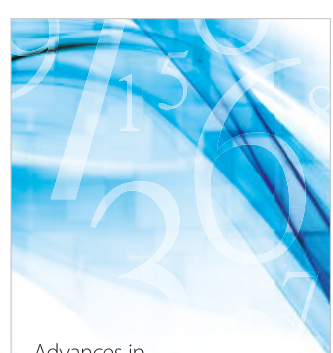

Advances in
Numerical Analysis
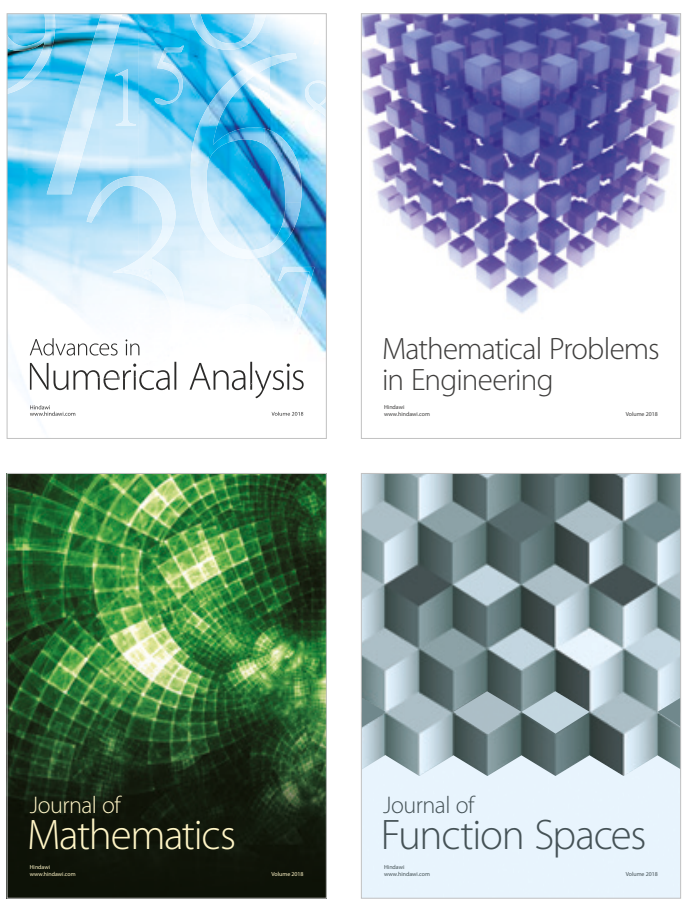

Mathematical Problems in Engineering

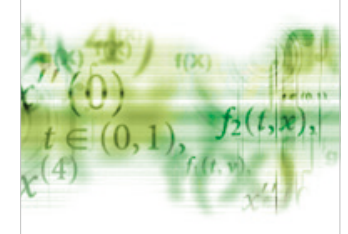

International Journal of

Differential Equations

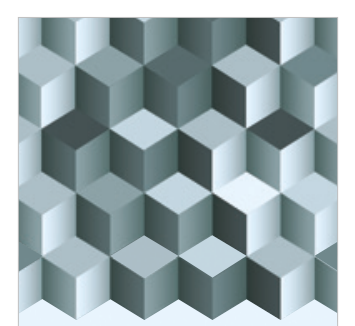

Journal of

Function Spaces

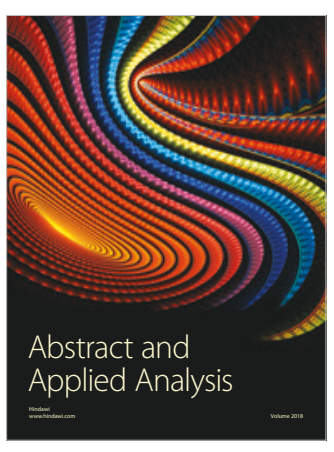

The Scientific

World Journal

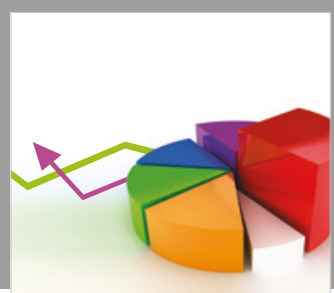

Journal of

Probability and Statistics
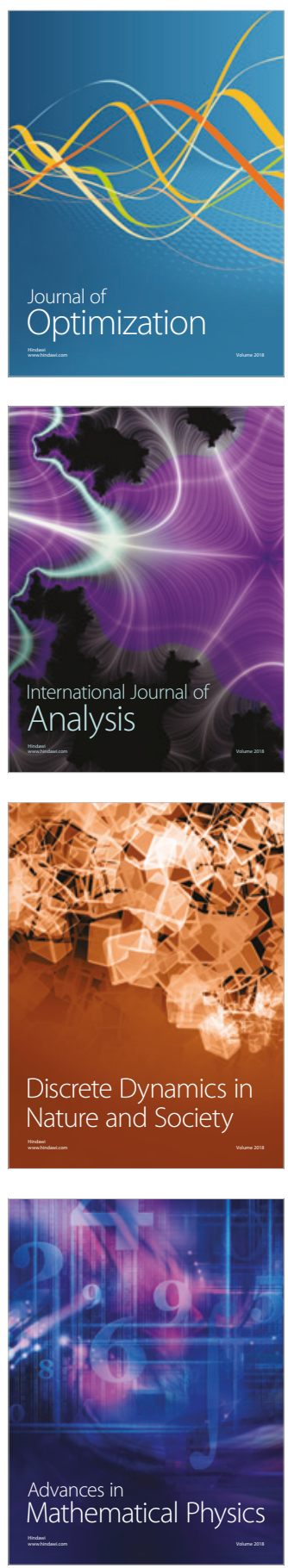